\title{
ANÁLISIS COMPARATIVO DE LA INFORMACIÓN DE TEMPERATURA DE LAS BASES DE DATOS WORLDCLIM, ECA\&D Y MODIS EN EL PALEÁRTICO OCCIDENTAL
}

\author{
Jorge Alonso-Carné \\ Departamento de Geografía y Ordenación del Territorio, \\ Facultad de Filosofía y Letras, Universidad de Zaragoza \\ C/ Pedro Cerbuna 12, 50009, Zaragoza \\ Departamento de Patología Animal, Facultad de Veterinaria, \\ Universidad de Zaragoza. C/ Miguel Servet 177, 50013, Zaragoza \\ Grupo GEOFOREST - Instituto Universitario de Ciencias Ambientales (IUCA), \\ Universidad de Zaragoza \\ jalonso@unizar.es \\ Agustín Estrada-Peña \\ Departamento de Patología Animal, Facultad de Veterinaria, \\ Universidad de Zaragoza. C/ Miguel Servet 177, 50013, Zaragoza \\ aestrada@unizar.es \\ Alberto García-Martín \\ Centro Universitario de la Defensa de Zaragoza, \\ Academia General Militar, Ctra. de Huesca s/n, 50090, Zaragoza \\ Grupo GEOFOREST - Instituto Universitario de Ciencias Ambientales (IUCA), \\ Universidad de Zaragoza \\ algarcia@unizar.es
}

Resumen: La proliferación y facilidad de acceso a grandes bases de datos de carácter científico tipo Open Data hace necesario un análisis sobre la adecuación de cada una de ellas a los objetivos perseguidos en cualquier trabajo de investigación o en la aplicación de un proceso de modelado. En este trabajo se comparan tres fuentes de datos validadas por comunidad científica y ampliamente utilizadas para el análisis de la temperatura 
(del aire y de superficie) a escalas medias (WorldClim, ECA\&D y MODIS) utilizando como área de estudio el territorio europeo al oeste de la frontera ruso-europea. Estas tres series de datos se comparan teniendo en cuenta dos tamaños de píxel y los distintos tipos de climas existentes. Como resultado, se verifica la existencia de diferencias entre estas series de datos, aunque no significativas, y se describen las limitaciones y funcionalidad de cada una de ellas.

Palabras clave: temperatura, MODIS, WorldClim, ECA\&D, Europa.

\begin{abstract}
The easy access to scientific Open Data large databases makes it necessary to analyze the adequacy of each of them to the objectives pursued in any research work or in the application of a modeling process. This paper compares three data sources validated by scientific community and widely used for the analysis of 2 types of temperature (air and land surface) at medium scales (WorldClim, ECA\&D and MODIS) using as study area the European territory west of the Russian-European border. These three datasets are compared taking into account two pixel sizes and different types of climates. As a result, we verified the existence of differences, although not significant, and described the limitations and functionality of each of these series.
\end{abstract}

Keywords: temperature, MODIS, WorldClim, ECA\&D, Europe.

\title{
1. Introducción
}

La temperatura es una de las variables climáticas más importantes dado que los cambios de magnitud y de la tasa de cambio de ésta pueden tener efectos importantes en la distribución de los organismos, en los equilibrios de los procesos ecológicos y, en consecuencia, en las actividades socioeconómicas (Geiger, 1965; Walther et al., 2002; Salinger, 2005; Zhao et al., 2005; Stern, 2006).

Dada su gran complejidad, las formas de variabilidad del clima son múltiples, con lo que pronosticarlas es una tarea difícil (Landa et al., 2008). No obstante, ante un escenario de cambio climático aceptado por el conjunto de las ciencias ambientales (Biología, Ciencias Agrarias, Geografía, Veterinaria...) resulta de gran interés la estimación con precisión de cualquiera de las variables que configuran un tipo de clima (Bustamante, 2003, Araujo et al., 2007).

Desde mediados de los años 90 existe una creciente disponibilidad de datos de distintas variables ambientales gracias a (i) el auge y desarrollo de las ciencias experimentales (estadística, informática...) y herramientas tecnológicas (Sistemas de Infor- 
mación Geográfica -SIG-, teledetección, programación); y (ii) la facilidad de acceso a la información gracias Internet y a las nuevas políticas de acceso libre para la investigación científica, sobre todo en áreas de interés global socio-ambiental relacionadas con el cambio climático (Patz et al., 2005; Field, 2012; McLeman, 2013, Sterling et al., 2013; Fordham et al., 2013). Estos avances están permitiendo desarrollar un abundante trabajo científico en el conjunto de las ciencias ambientales.

Centrándonos en la variable temperatura, las dos fuentes primarias de datos utilizadas en la literatura científica revisada son: (i) las estaciones meteorológicas, que capturan la temperatura del aire a una altura de $2 \mathrm{~m}$; y (ii) la teledetección, que captura la temperatura de superficie.

Las estaciones meteorológicas son el sistema tradicional de captura de la temperatura del aire vigente desde el auge de la climatología a finales del Siglo XVIII. Desde entonces, la disponibilidad, continuidad y calidad de las series de datos proporcionadas por este sistema ha ido creciendo, siendo para ello fundamental el papel de la Organización Mundial de la Meteorología WMO (www.wmo.ch). El desarrollo en los últimas tres décadas de los SIG y de la estadística permite interpolar, normalizar y desarrollar mapas de temperatura a partir de la información puntual de estas estaciones aplicando distintos algoritmos cada vez más complejos como, por ejemplo, Superficies de Tendencia, lineales/aditivos Generalizados, Regresión Sencilla, Múltiple, GARP, Krigging y Max. Entropía (Bustamante, 2003; Haylock et al., 2008). Dos de las bases de datos climáticas con datos de temperatura del aire procedentes de estaciones meteorológicas más usadas en investigación son WorldClim y ECA\&D. WorldClim (Hijmans et al., 2005; <http://www. WorldClim.org>) ofrece datos a nivel mundial desde el año 1960 con una periodicidad mensual en formato ráster con una resolución máxima de $1 \mathrm{~km}^{2}$ (de 30 arc segundos a 10 minutos) a partir de una red mundial de más de 14.000 estaciones de datos a través de un spline de placa delgada ANUSPLIN (Hutchinson, 1997). Esta base de datos climática ha sido utilizada como referencia en el desarrollo de un gran número de estudios ambientales al ser la primera que ofreció a escala mundial esta información (p.e.: Ríos Muñoz et al., 2009; Rochlin et al., 2013). Por su parte ECA\&D (Haylock, 2008; www.ecad.knmi.nl) ofrece datos climáticos a escala Pan-Europea desde 1950, con una red en constante crecimiento que ya supera las 6.000 estaciones (Holanda, Irlanda y Suiza poseen la mayor densidad, Escandinavia, España, África y Balcanes la inferior). Ofrece datos a 4 escalas: 0.25 ó 0.5 grados en coordenadas geográficas y 0.22 ó 0.44 en coordenadas Rotated Pole. El algoritmo utilizado es complejo y se desarrolla en 3 fases integrando un spline de Placa Delgada "Thin-Plate" y un ajuste por Krigging a nivel diario y mensual (ECA\&D\&KNMI, 2008 y 2013). Su uso está en auge asociado a su desarrollo siendo su utilización para analizar la temperatura también abundante en los últimos años (p.e.: Sánchez-Lorenzo et al., 2008; Van der Schrier, 2013).

En cuanto a la captura de la temperatura de superficie por parte de la teledetección, esta se basa en el uso de la radiación del área de estudio adquirida por los sensores en 
el espectro térmico y la aplicación de distintos algoritmos para su conversión en información de interés climático-ambiental. Desde 1978, con el lanzamiento de Landsat 3, se disponen de datos de temperatura de superficie con una resolución espacial de, al menos, 240 m (Chuvieco, 1996). Centrándonos exclusivamente en esta variable, cabe destacar de la importancia del proyecto MODIS que, compuesto por dos satélites (Terra, lanzado en el año 2000 y Aqua en el año 2002) constituyó la primera misión específicamente desarrollada para la observación y el estudio de la superficie terrestre y el análisis del cambio climático ofreciendo desde entonces cada 1-2 días dos datos de temperatura de superficie diurna y nocturna con una resolución de $0.05^{\circ}$ en el ecuador.

Temperatura del aire y temperatura de superficie son dos variables distintas de temperatura, pero que se encuentran altamente correlacionadas entre sí. Por ello es habitual integrar ambas variables para optimizar espacialmente la variable temperatura en su uso en modelos de procesos ecológicos (Sun, et al., 2005; Liu S. et al., 2016).

El auge de la disponibilidad de datos no debe hacer olvidar que en todo caso siempre se debe de considerar la validez y adecuación de los mismos para el problema o proceso que se quiere conocer o modelizar. Esto es especialmente relevante a la hora de seleccionar cualquier variable climática dada la condición de éstas como variables de base para la práctica totalidad de estudios ambientales. Así, resulta imprescindible una buena valoración y conocimiento de la precisión, cantidad, proyección y escala de la fuente de datos a utilizar de cara a lograr unos resultados significativos y una correcta interpretación de los mismos. En este sentido, el conocido como "efecto mariposa" (Lorenz, 1963, Dantas-Torres, 2015), explica como pequeñas variaciones en los parámetros de un modelo de predicción provocan resultados muy diferentes y como de compleja y limitada es toda propuesta de predicción a largo plazo en este campo. La captación de los valores más exactos posibles de la temperatura dada su continuidad espacio-temporal y su implicación en procesos que se suceden a distintas escalas espaciales y temporales, se convierte por tanto es un proceso clave para el éxito de cualquier estudio ya sea de monitorización o predicción ambiental.

\section{2. Área de estudio}

El área de estudio incluye la zona del Paleártico occidental incluida entre los $18.5^{\circ} \mathrm{W}$,

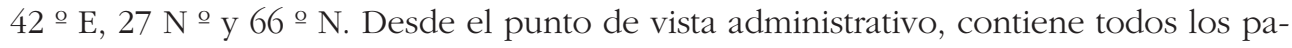
íses europeos al Oeste de la frontera ruso-europea por lo que, desde el punto de vista climático, se recoge toda la variabilidad climática presente en este continente. 


\section{Materiales y métodos}

El presente trabajo tiene como objetivo analizar las diferencias que existen en la variable climática temperatura a una escala media Europea a partir de varias de las series de datos de temperatura (de superficie y del aire) de uso más frecuente, asumiendo las diferencias temporales, espaciales y de origen entre las mismas en un contexto de cambio climático. La información de temperatura de superficie es proporcionada por el proyecto MODIS con datos normalizados a través de una transformada de Fourier que busca descartar los errores intrínsecos asociados a la captura y procesado de estos datos como el vapor de agua (Scharlemann et al., 2008) y WorldClim y ECA\&D son las bases de datos climáticas utilizadas basadas en interpolaciones a escalas medias de datos de temperatura del aire procedentes de estaciones meteorológicas.

En concreto, se analizan las variables temperatura máxima, media y mínima (TMax, TMedia, TMin) para la región Paneuropea a una escala homogeneizada teniendo en cuenta los dos tamaños de píxel de las fuentes (0.05ํㅡ y $0.25^{\circ}$ ) Además, de modo complementario, se compararon aplicando un análisis ANOVA los datos MODIS con los datos reales puntuales de quince estaciones meteorológicas de la red ECA\&D teniendo en cuenta los distintos tipos de clima existentes en el área de estudio según LandMap2 (Mücher et al., 2010).

\subsection{Datos MODIS: Terra y Aqua}

Los datos MODIS de la serie mensual MOD11C3 y MYD11C3 (versión 5) fueron descargados para el período comprendido entre los años 2000 y 2010 de la página web de este satélite (https://lpdaac.usgs.gov/). Estos productos ofrecen las mediciones de temperatura de superficie (TST), tanto de Terra como Aqua (este último desde 2002). Ambos satélites tienen diferentes tiempos orbitales, tanto en el área de estudio como en el resto del planeta (Wan et al., 2004). La resolución espacial es de un valor nominal de $0.05^{\circ}$ en el Ecuador (5.600 m, aproximadamente). Los datos de la versión $5 \mathrm{de}$ MODIS son validados en su producción por el propio equipo científico de MODISNASA, lo que se traduce en que estos datos son válidos para su uso en publicaciones y estudios científicos (Wan, 2007).

Para cada período de 30 días del intervalo 2000-2010 en el caso de Terra y 2002-2010 en el caso de Aqua se han utilizado las pasadas de "día" y "noche". Las imágenes fueron recortadas a los límites geográficos de la región de estudio y se transformaron para obtener la medición de la temperatura en grados Celsius (o) mediante la utilización del software de Sistemas de Información Geográfica (SIG) GRASS (https://grass.osgeo.org/). Seguidamente, haciendo uso de las banderas de calidad que aporta MODIS, se filtraron las imágenes descartando aquellos pixeles calificados como nulos, no validos y los 
valorados como afectados gravemente por errores de estimación $\left(>3^{\circ} \mathrm{C}\right.$, en este caso, son principalmente aquellos en donde la presencia de nubes, nieve o agua alteran el correcto funcionamiento del algoritmo de MODIS). Una vez procesados y optimizados los datos, se obtuvieron los datos de media de las tres variables en cada mes del año, los cuales fueron procesados mediante una transformada de Fourier para descartar la presencia de vapor de agua (Scharlemann et al., 2008). Posteriormente para su comparación con el resto de series de datos utilizadas en este trabajo, se obtuvo con GRASS el mapa anual medio del período de estudio de cada una de las tres variables estudiadas y se calculo el porcentaje de error relativo de las comparaciones resultantes. Asimismo, para permitir su comparación con la información de la base ECA\&D, también se elaboraron mapas con píxeles a $0.25^{\circ}$ de resolución mediante ajuste bilineal con GRASS.

\subsection{Datos WorldClim}

Los datos de WorldClim fueron obtenidos de su página web oficial (http://www. WorldClim.org/), descargándose temperatura máxima, media y mínima a 5 arco-minutos de resolución para los 12 meses del año. Estos datos están validados para su aplicación en usos científicos (Hijmans et al., 2005). En su actual versión (v.1.4) esta base de datos ofrece información de temperatura para la serie temporal 1960-1990, existiendo en fase beta de desarrollo su versión 2, que ofrecerá datos de la serie temporal 19702000, (http:// WorldClim.org/version2). Los datos descargados fueron integrados en GRASS desde donde se realizó un proceso de tratamiento de los mismos, descartando "outliers" o datos extremos que se reconocieron como no validos (principalmente zonas heladas, montañas o de agua) para finalmente obtener datos anuales promedio de las 3 variable de estudio. Para su comparación con ECA\&D, de igual modo que en el caso de los datos MODIS, se derivaron mapas con píxeles a $0.25^{\circ}$ de resolución mediante ajuste bilineal con GRASS. Además se comprobó la suma total de error de cada imagen comparativa resultante para valorar sus diferencias con el resto.

\subsection{Datos ECA\&D}

Los datos de ECA\&D se obtuvieron de la página web oficial del proyecto (http:// www.ecad.eu/). Se descargaron los datos de temperatura máxima, media y mínima a 0.25ํํㄹ de resolución para las siguientes series temporales: 1950-2010, 1960-1990, 20002010 y 2002-2010 de forma que se recogieron datos de toda la serie temporal disponible y las coincidentes para favorecer su comparación por pares a WorldClim y MODIS (Terra y Aqua). Estos datos se integraron y procesaron de igual forma en GRASS en donde se obtuvieron los mapas resultantes de temperaturas promedio para cada variable y serie de tiempo. Se comprobó la suma total de error de cada imagen comparativa resultante para valorar sus diferencias con el resto. 


\subsection{Estaciones control}

Al objeto de tener unos datos de validación de cada uno de los climas presentes en el territorio europeo según LandMap2 Level 1 (Mücher et al., 2010), se seleccionaron 14 estaciones de control en la base de datos ECA\&D para las tres variables estudiadas, teniendo al menos una estación por cada tipo de clima (ver Tabla 1). Como se puede observar en la Figura 1, la selección de estas estaciones busca garantizar una buena distribución espacial de las variables estudiadas recogiendo toda la variabilidad de la zona de estudio.

Tabla 1. Relación de climas existentes en cada estación de control y su ubicación

\begin{tabular}{|l|l|c|c|c|c|}
\hline Clima & Estación & País & $X$ & $Y$ & Altitud (m) \\
\hline Continental & Augsburg & DE & 10,94 & 48,43 & 461,00 \\
\hline Atlántico & Birr & IE & $-7,88$ & 53,09 & 70,00 \\
\hline Mediterráneo & Córdoba & ES & $-4,85$ & 37,85 & 90,00 \\
\hline Estepario & Doneck & UA & 37,80 & 48,10 & 259,00 \\
\hline Atlántico & Esksalenmir & GB & $-3,20$ & 55,32 & 242,00 \\
\hline Alpino & Teigarhorn & IS & $-15,23$ & 64,68 & 22,00 \\
\hline Boreal & Jyvaskula & FI & 25,68 & 62,40 & 137,00 \\
\hline Mediterráneo & Milán & IT & 9,19 & 45,47 & 150,00 \\
\hline Boreal & Minsk & BY & 27,53 & 53,87 & 222,00 \\
\hline Continental & Novisad & RS & 19,85 & 45,33 & 84,00 \\
\hline Atlántico & Porto & PT & $-8,60$ & 41,13 & 93,00 \\
\hline Anatolia & Sivas & TR & 37,02 & 39,75 & 1285,00 \\
\hline Alpino & SonnBlick & AT & 12,95 & 47,05 & 3106,00 \\
\hline Mediterráneo & Toulouse & FR & 1,38 & 43,62 & 151,00 \\
\hline
\end{tabular}

\subsection{Análisis comparativo de los datos}

El trabajo de análisis comparativo de los datos ofrecidos por estas tres series se realizó a tres niveles: (i) análisis comparativo por pares de la media de las tres variables estudiadas (TMax, TMedia y TMin) de las tres bases de datos utilizadas (MODIS, WorldClim 
y ECA\&D) para las escalas temporales (1950-2010, 1960-1990, 2000-2010 y 2002-2010) y espaciales $\left(0.05^{\circ}\right.$ y $\left.0,25^{\circ}\right)$ consideradas; (ii) comparación del valor real conocido en los 14 puntos de control seleccionados en el apartado 3.4. con el valor de temperatura proporcionado por las series de datos en cada escala espacial y temporal utilizada y análisis estadístico ANOVA para comprobar diferencias significativas entre estas; y (iii) comparación del valor medio de cada una de las tres variables para las escalas temporales y espaciales delimitadas para cada clima de LandMap2 Level 1 (Mücher et al., 2010) de cara a facilitar relaciones ecológicas en la explicación de los resultados.

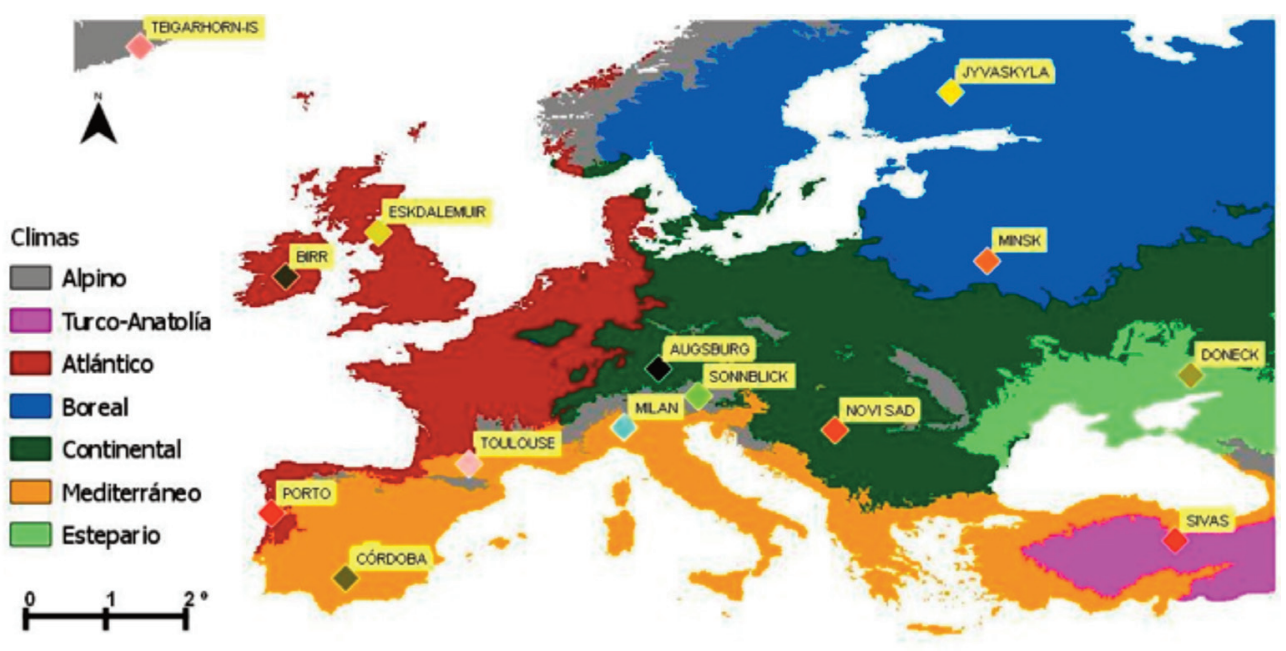

Figura 1. Relación de climas Landmap2 Level 1 en el área de estudio y estaciones de control seleccionadas.

\section{Resultados}

\subsection{Análisis comparativo entre las distintas fuentes de datos}

\subsubsection{MODIS- WorldClim}

Los resultados (Figura 2) muestran que existe un alto grado de coincidencia (diferencias entre $-1^{\circ} \mathrm{C} /+5^{\circ} \mathrm{C}$ y la mediana de todas las series inferior a $1^{\circ} \mathrm{C}$ de diferencia) en latitudes medias para la TMax entre MODIS y WorldClim (mayor igualdad con el satélite Terra que con Aqua), existiendo un gradiente de diferencia positiva hacia el sur (diferencias de hasta $+15^{\circ} \mathrm{C}$ en Terra y $+20^{\circ} \mathrm{C}$ en Aqua) y negativo hacía el norte (hasta $-5^{\circ} \mathrm{C}$ ). La diferencia global es 2,99 veces superior entre Aqua y Worldclim que con respecto a Terra. 

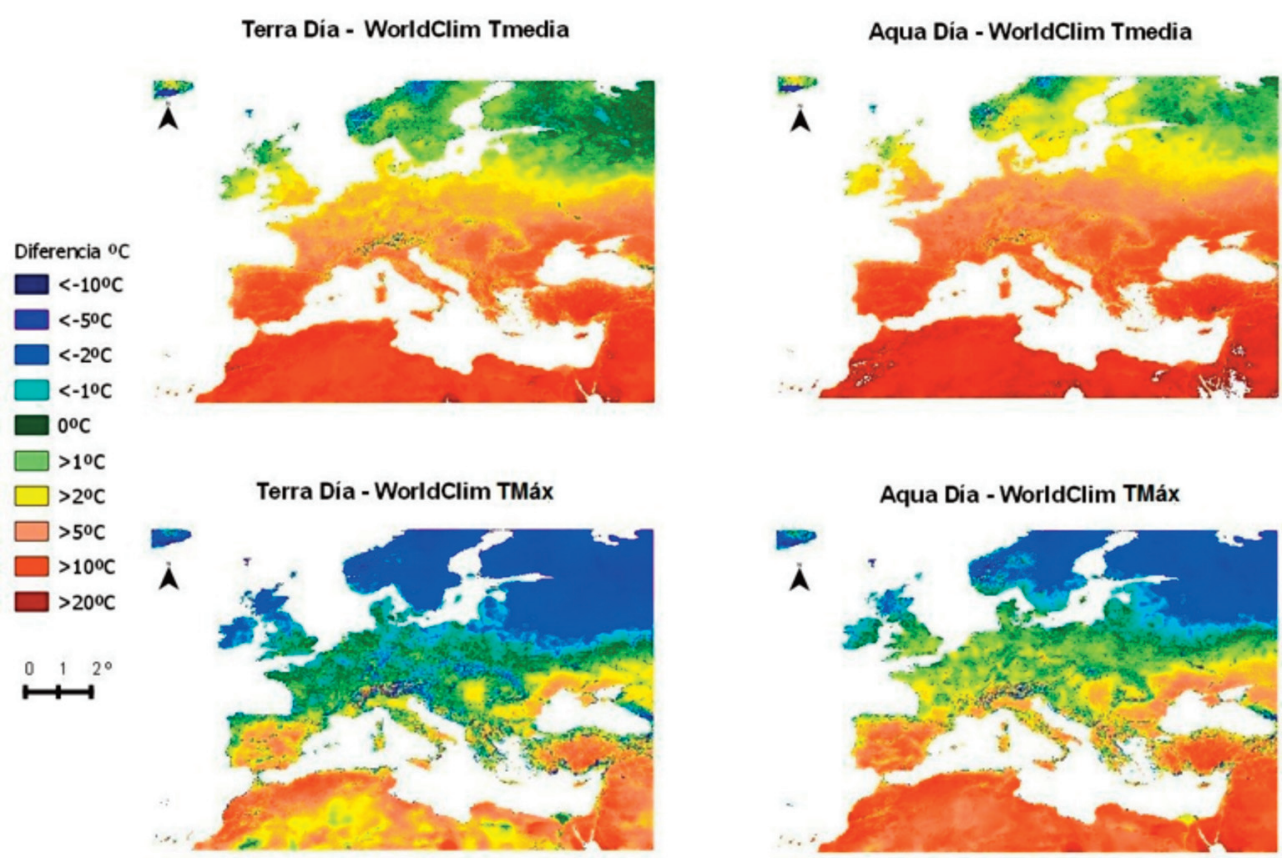

Figura 2. Comparación datos de MODIS (Terra y Aqua) con datos de WorldClim para TMax y TMedia.

Respecto a la TMedia, solo existe una buena relación (entre 0 y $2^{\circ} \mathrm{C}$ de diferencia) entre MODIS y WorldClim en la zona de Escandinavia por encima del paralelo 55으, siendo los valores de temperatura de MODIS siempre superiores en el resto del territorio estudiado (la mediana de las series son de $3,87^{\circ} \mathrm{C}$ y $5,4^{\circ} \mathrm{C}$ ). La diferencia global de WorlClim es 1,32 veces mayor con respecto a Aqua que con respecto a Terra.

En cuanto a la TMin, existe una clara mejor relación de WorldClim con Terra que con Aqua (Figura 3). Terra presenta una buena coincidencia en torno a $\pm 2^{\circ} \mathrm{C}$ en todo el territorio salvo zonas montañosas, Islandia y ciertos puntos de la costa atlántica. En el caso de Aqua presenta una relación negativa superior a los $5^{\circ} \mathrm{C}$ generalizada entre ambas fuentes siendo solo coincidente en ciertas zonas del Mediterráneo. La diferencia global es 7,49 veces mayor entre WorldClim y Aqua que con respecto a Terra. En este caso la compensación de errores hace que las medianas de las series se sitúen sin embargo en términos semejantes para Aqua $\left(-2.77^{\circ} \mathrm{C}\right.$ y $\left.-2,89^{\circ} \mathrm{C}\right)$ y para Terra en $\left(-3,15^{\circ} \mathrm{C}--3,74^{\circ} \mathrm{C}\right)$. 

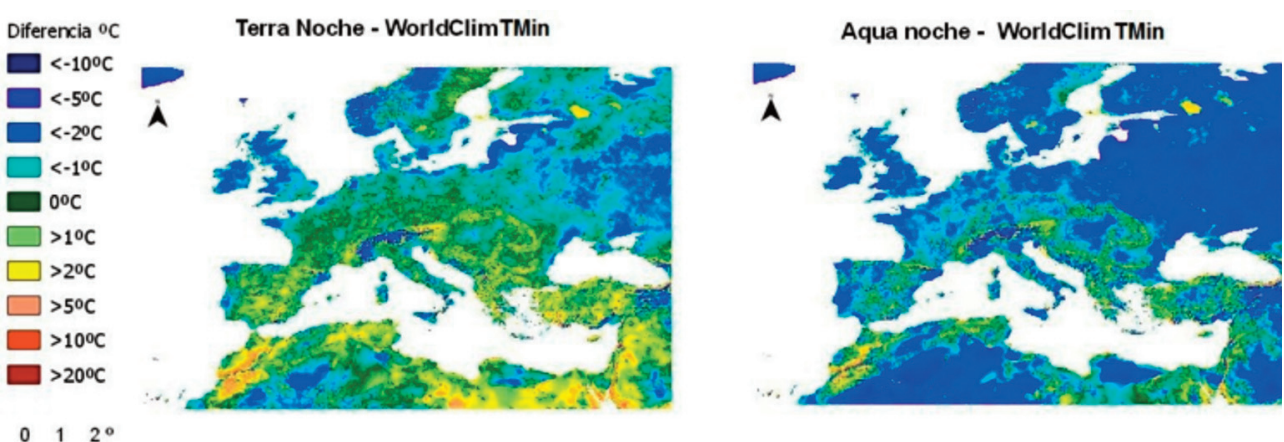

Figura 3. Comparación MODIS (Terra y Aqua) con datos de WorldClim para la TMin.

\subsubsection{MODIS - ECA\&D}

El patrón es similar a la relación MODIS- WorldClim observado en el anterior sub-apartado, dado que los valores de TMax tienen una buena coincidencia $\left(-5^{\circ} \mathrm{C} / 5^{\circ} \mathrm{C}\right)$ en latitudes medias tanto en Terra (Figura 4) como en Aqua (Figura 5), aunque en una menor franja espacial, existiendo de igual forma un gradiente positivo hacía el sur y negativo hacía el norte.

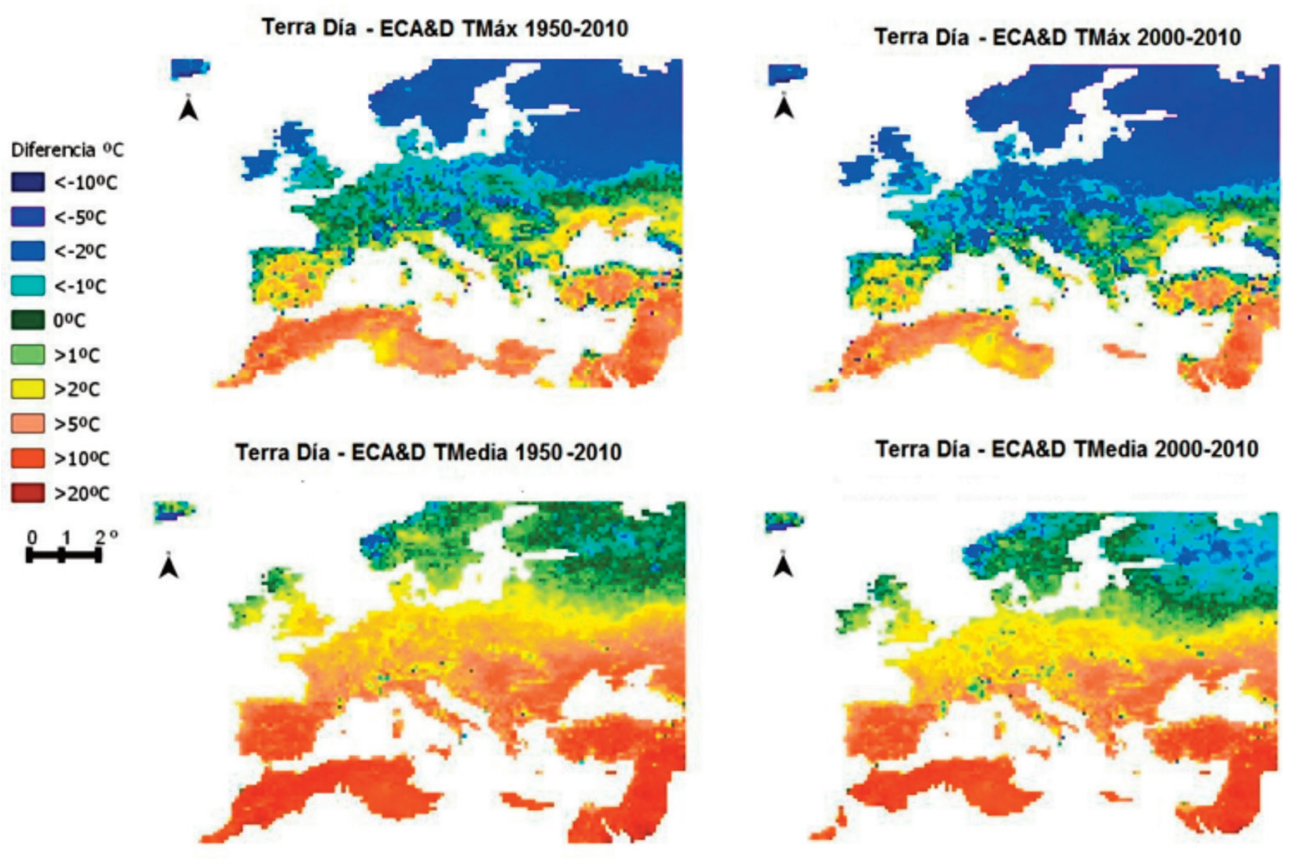

Figura 4. Comparación de MODIS Terra con ECA\&D para TMax y TMedia para las series $1950-2010$ y 2000-2010. 

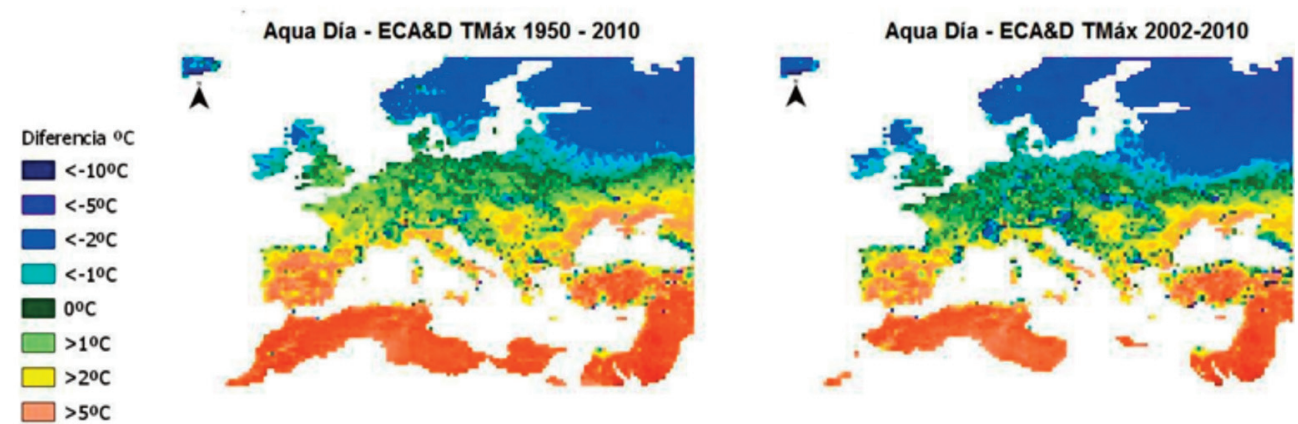

Aqua Dia - ECA\&D TMedia 1950 - 2010

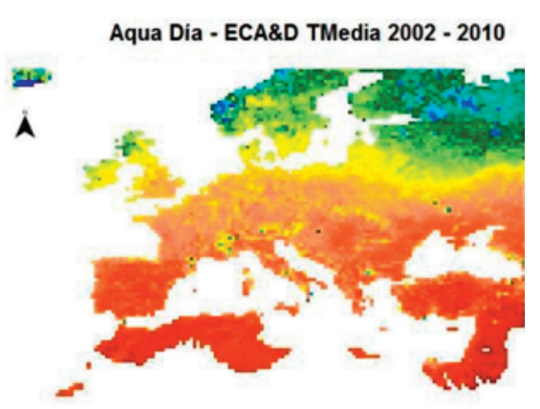

Figura 5. Comparación de MODIS Aqua con ECA\&D para temperatura máxima y media para las series 1950-2010 y 2002-2010.

En el caso de la variable TMedia, se repite también el patrón descrito anteriormente, dada la buena relación en latitudes por encima del paralelo $55^{\circ} \mathrm{N}$ y un gradiente positivo hacía el sur. La desigualdad entre ambos datos tiene una clara correlación espacial siendo proporcionalmente inferior a WorldClim en latitudes medias.

La comparación de ambas series refleja que para los datos de Terra el ajuste es ligeramente mejor en la serie completa de 1950-2010, mostrando ECA\&D datos más elevados para la zona de estudio en el caso de 2000-2010 (1,04 para el conjunto). En el caso de Aqua, la serie 2002-2010 ajustan mejor respecto la TMax (0,91 respecto 1950-2010) y 1950-2010 (0,72 respecto de la diferencia global de 2002-2010) en el caso de la TMedia.

En cuanto a la TMin, los datos reflejan una mayor concordancia entre Terra y ECA\&D que Aqua (Figura 6). Los datos son coincidentes en el entorno del mediterráneo y áreas centrales de la zona de estudio (Suecia, Alemania y ExYugoslavia) mostrando ECA\&D en el resto de los casos datos superiores a MODIS. En la comparación Terra-ECA\&D de la serie 1950-2010 presenta una diferencia menor $(0,96)$ respecto de la serie 20022010. En Aqua es al revés, siendo esta relación de 1,02. 


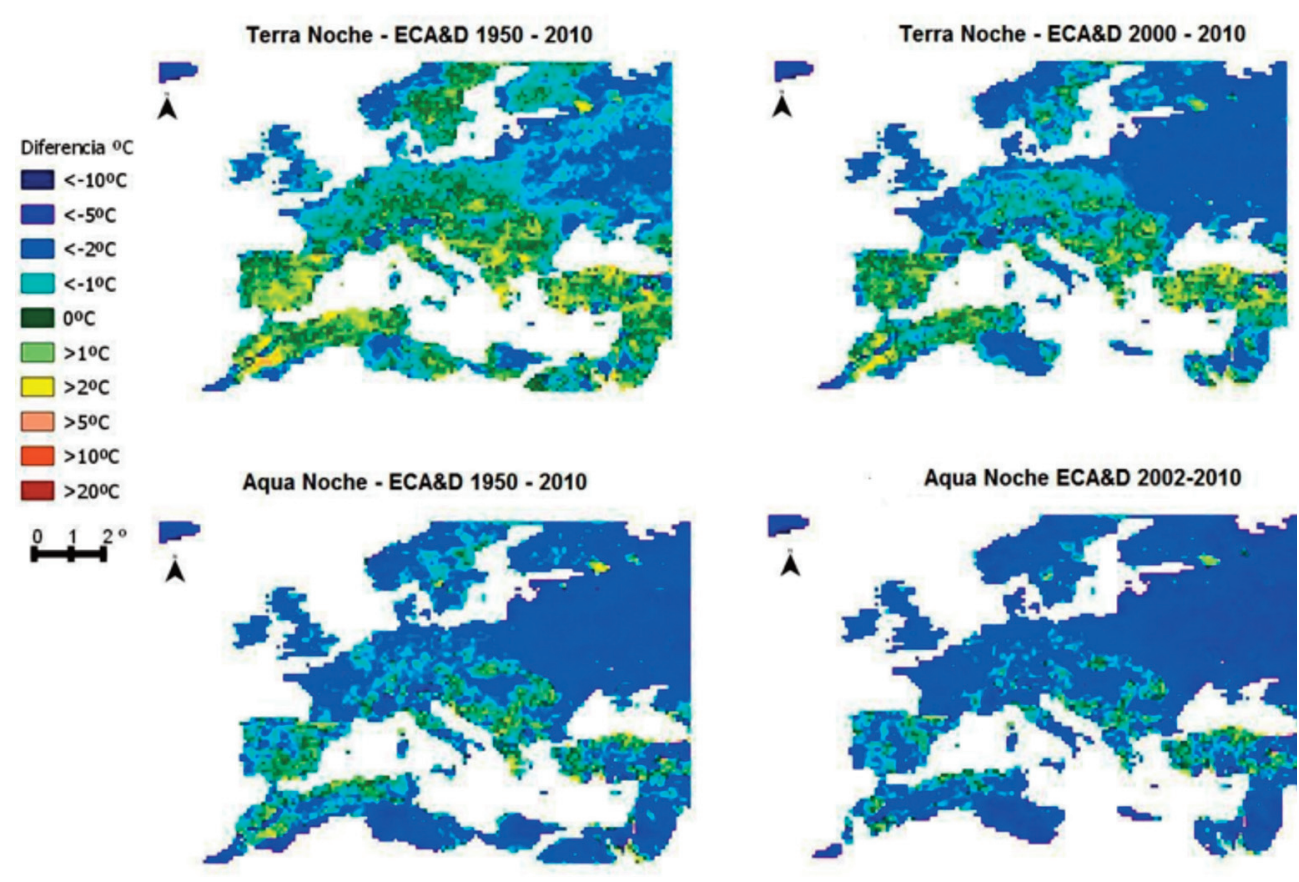

Figura 6. Comparación de datos MODIS Terra y Aqua con ECA\&D para

la TMin para las series 1950-2010, 2000-2010 y 2002-2010.

\subsubsection{ECA\&D - WorldClim}

Los comparación de ambas bases de datos reflejan resultados semejantes con una escasa diferencia de +/- $1^{\circ} \mathrm{C}$ tanto en la TMax como en la TMedia para casi todo el territorio. Solo se identifican márgenes mayores $\left(+/-2^{\circ} \mathrm{C}\right)$. en las zonas más al sur y al norte de la zona de estudio, así como las regiones montañosas (ver Figura 7). Como era de esperar, las diferencias son menores para la comparación de series coincidentes, es decir, para la de 1960-1990 (la diferencia total entre mapas es 12 veces mayor en el caso de la serie 1950-2010 para la TMax y 6,3 veces mayor para la TMedia).

En cuanto a la TMin, existe una gran coincidencia entre ECA\&D y WorldClim (Figura 8) (mediana de las series de datos con una diferencia $<0,1^{\circ} \mathrm{C}$ en ambos casos), siendo superior, como, en el caso anterior, en la serie 1950-2010 (6,34 veces). Las mayores diferencias se focalizan en las zonas montañosas, en el entorno del Mediterráneo y en las latitudes más al norte. Todas las diferencias oscilan en $+/-2^{\circ} \mathrm{C}$. 
ECA\&D TMáx - WorldClim TMáx 1960 -1990

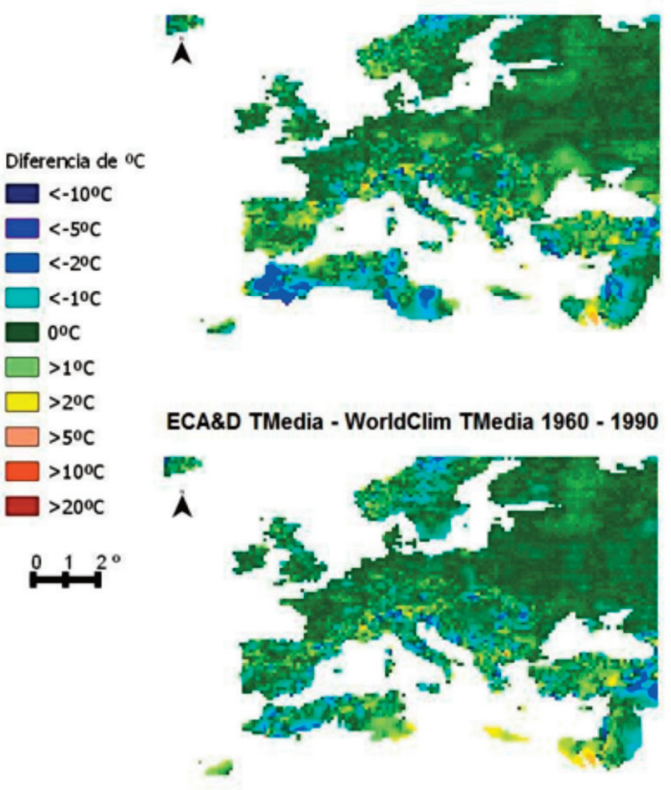

ECA\&D TMáx - WorldClim TMáx 1950-2010

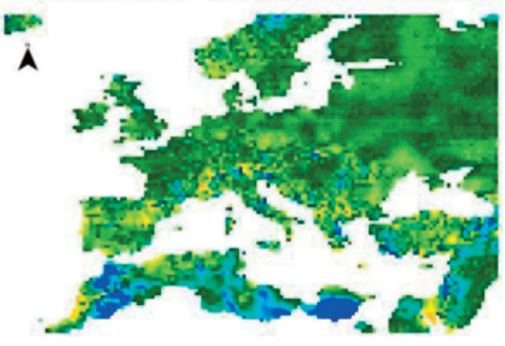

ECA\&D TMedia - WorldClim TMedia 1950-2010

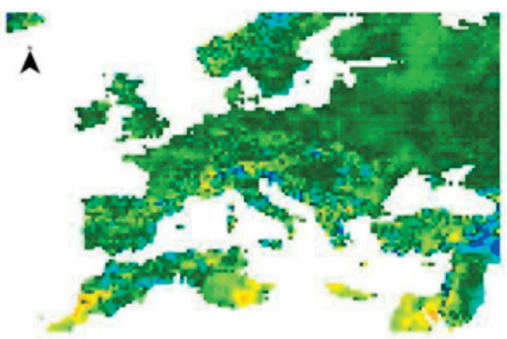

Figura 7. Comparación de datos ECA\&D con WorldClim para la TMax y TMedia para las series 1960-1990 y 1950-2010.
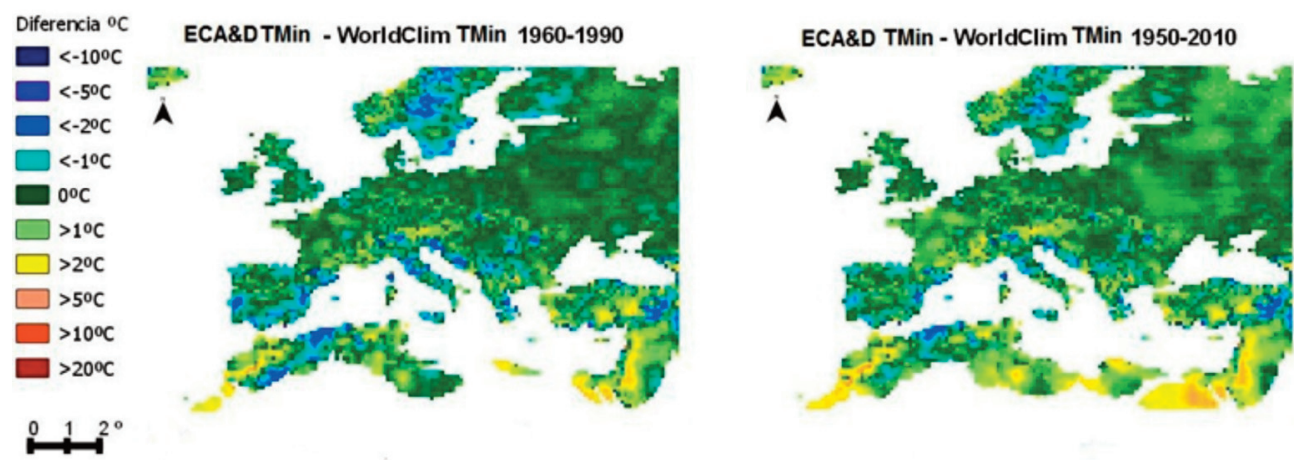

Figura 8. Comparación de datos ECA\&D con WorldClim para la TMin para las series 1960-1990 y 1950-2010. 


\subsection{Comparación de los datos MODIS, WorldClim y ECA\&D con las estaciones control}

\subsubsection{Análisis estadístico de las series de datos de las estaciones control}

Cuando se comparan las series de temperatura de acuerdo a los puntos de control se comprueba que la media y la varianza de todas las series son coincidentes para las tres variables a estudio (TMax, Figura 9; TMedia, Figura 10; y TMin, Figura 11). Los análisis ANOVA y Leneve constatan que no hay diferencias significativas respecto a la media y la varianza de ninguna de las series para ninguna de las tres variables ( $p>0,7$ y $p>0,35$, respectivamente). En todos los casos se observa que con un intervalo de confianza del 95\% todas las series se mueven en un rango similar de datos, siendo el rango de los datos de MODIS en las tres variables superior al aportado por ECA\&D y WorldClim. Dicha similitud es coherente a la utilidad científica de los mismos.

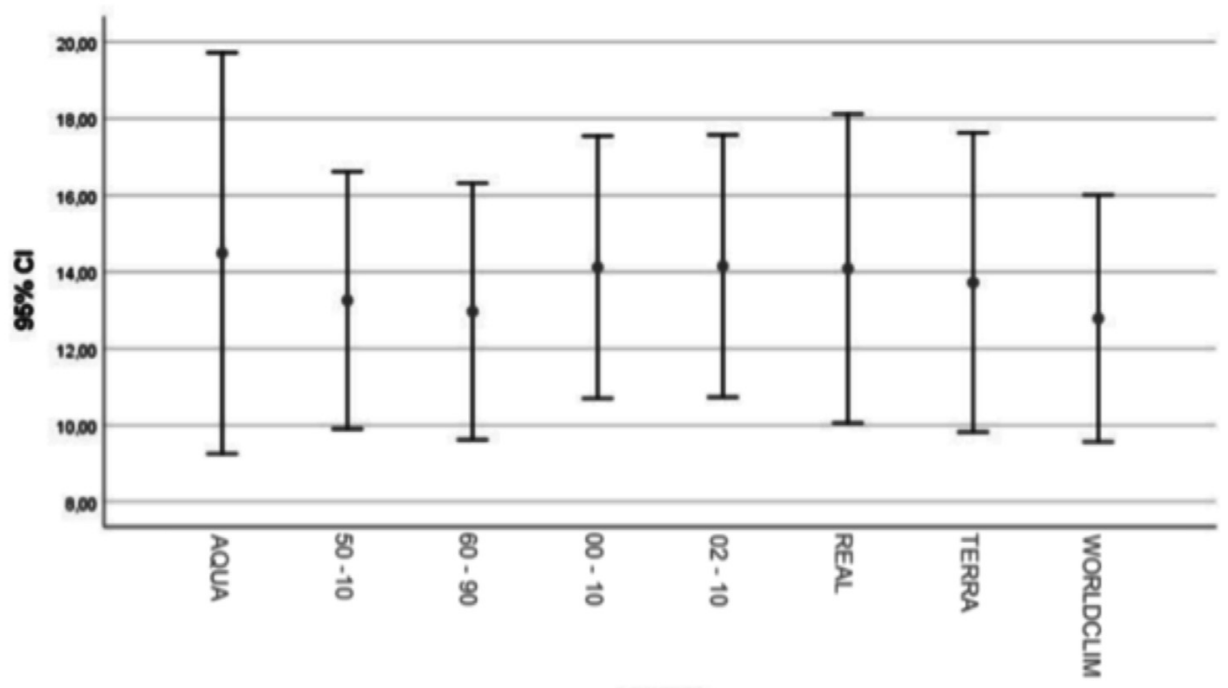

Figura 9. Gráfico de barras de error con un intervalo de confianza del 95\% para TMax.

Respecto a la Tmax, el rango de datos ajusta de forma precisa respecto a su mínimo, teniendo todas las series su valor promedio en un rango de $+/-1^{\circ} \mathrm{C}$, ajustando también de forma bastante precisa respecto a su máximo salvo en el caso de MODIS, destacando dentro de esto los datos de Aqua, que sobrepasa dicho rango ( $1^{\circ}$ grado en el mínimo y $2^{\circ} \mathrm{C}$ en el máximo). 


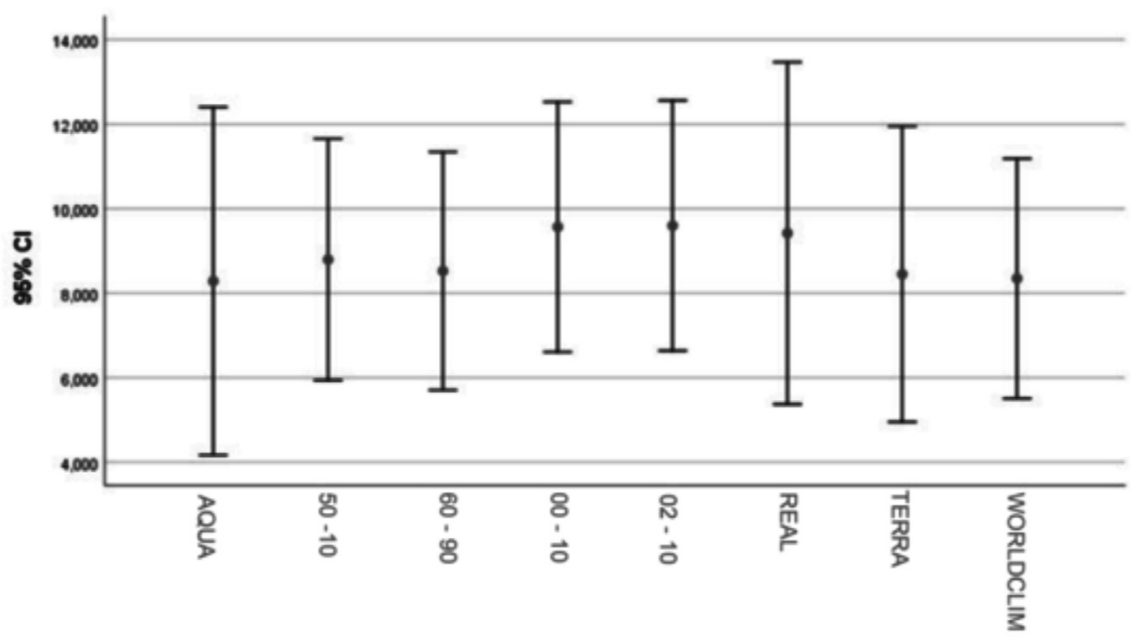

Figura 10. Gráfico de barras de error con un intervalo de confianza del 95\% para TMedia.

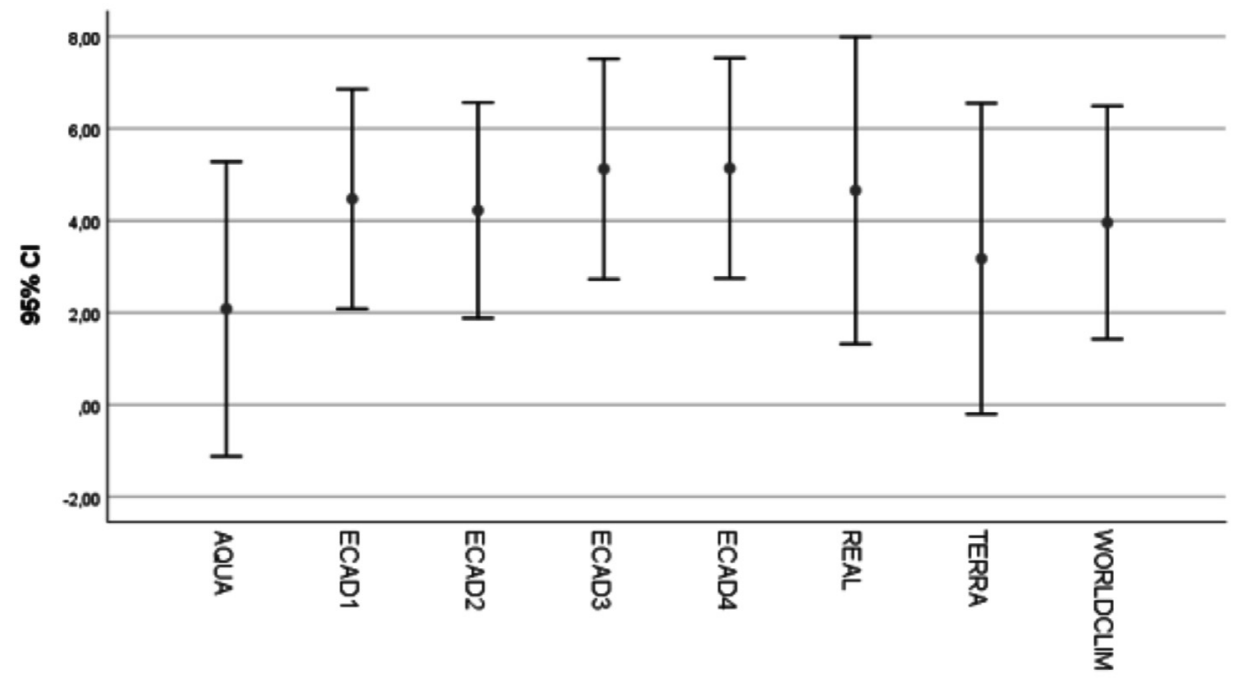

Figura 11. Gráfico de barras de error con un intervalo de confianza del 95\% para TMin. 
La TMedia presenta más diferencias. Se comprueba que el rango de las series de ECA\&D y WorldClim se sitúa en su conjunto dentro del rango real de datos aunque no abarcan su conjunto. En el caso de los datos de MODIS sobrepasan sus valores mínimos $\left(<2^{\circ} \mathrm{C}\right)$ y, como en el resto de casos, no alcanza sus valores más altos de rango.

Por último, los datos de TMin presentan tendencias similares. ECA\&D recoge toda su serie dentro de la serie de datos reales, no abarcando el conjunto de su rango. WorldClim ajusta de igual forma casi en su totalidad con el rango real de datos, sobrepasando sus valores mínimos por un escaso margen $\left(<1^{\circ} \mathrm{C}\right)$. Al igual que pasaba en la variable TMedia, ECA\&D y WorldClim no alcanzan a recoger los datos más altos de temperatura de la serie de temperatura real. Las variables de MODIS no alcanzan los valores altos del rango de datos reales y, en cambio, vuelven a superar ampliamente su mínimo (Aqua $>2{ }^{\circ} \mathrm{C}$ ).

\subsubsection{Análisis de la temperatura máxima (TMax) por estaciones}

En el caso de la variable TMax, salvo en el caso de Islandia y en la zona de los Alpes, las diferencias encontradas entre las tres bases de datos utilizadas y las estaciones de control se sitúan en torno a un máximo de $5^{\circ} \mathrm{C}$ dispersándose las diferencias hasta los $7^{\circ} \mathrm{C}$ e incluso $13^{\circ} \mathrm{C}$ en las zonas heladas y de montaña capturadas mediante $\mathrm{MO}-$ DIS (Figura 12 y Tablas 2 y 3).

Un análisis más en profundidad muestra como las Series de datos de ECA\&D y WorldClim, al estar correlacionadas, presentan datos semejantes, presentando tendencia a ofrecer datos más elevados en las series más recientes, sea por una menor cantidad de datos para realizar el promedio, sea asociado al fenómeno de cambio climático. Por su parte, MODIS presenta mayores divergencias en las estaciones más al norte y de carácter montañoso, obteniendo buenos resultados en el resto. En cuanto a las diferencias entre los dos satélites que componen esta base de datos, señalar que Aqua presenta siempre datos superiores a los de Terra, siendo esta situación sobre todo visible en el clima mediterráneo.

\subsubsection{Análisis de la temperatura media (TMedia) por estaciones}

Como se observa en la Figura 13 y Tablas 2 y 3, en el caso de la TMedia se repiten las mismas tendencias que con las TMax, existiendo problemas con las estaciones de Islandia y los Alpes. Los datos de MODIS se alejan de los datos de TMedia por más de $5^{\circ} \mathrm{C}$ en todos los casos, salvo en las estaciones de control situadas a mayor latitud. Los datos de WorldClim reflejan siempre un valor inferior al dato real de la serie ECA\&D. Centrándonos en esta última base de datos, se muestra que las temperaturas medias son más suaves cuantos más son los años de referencia tomados. 

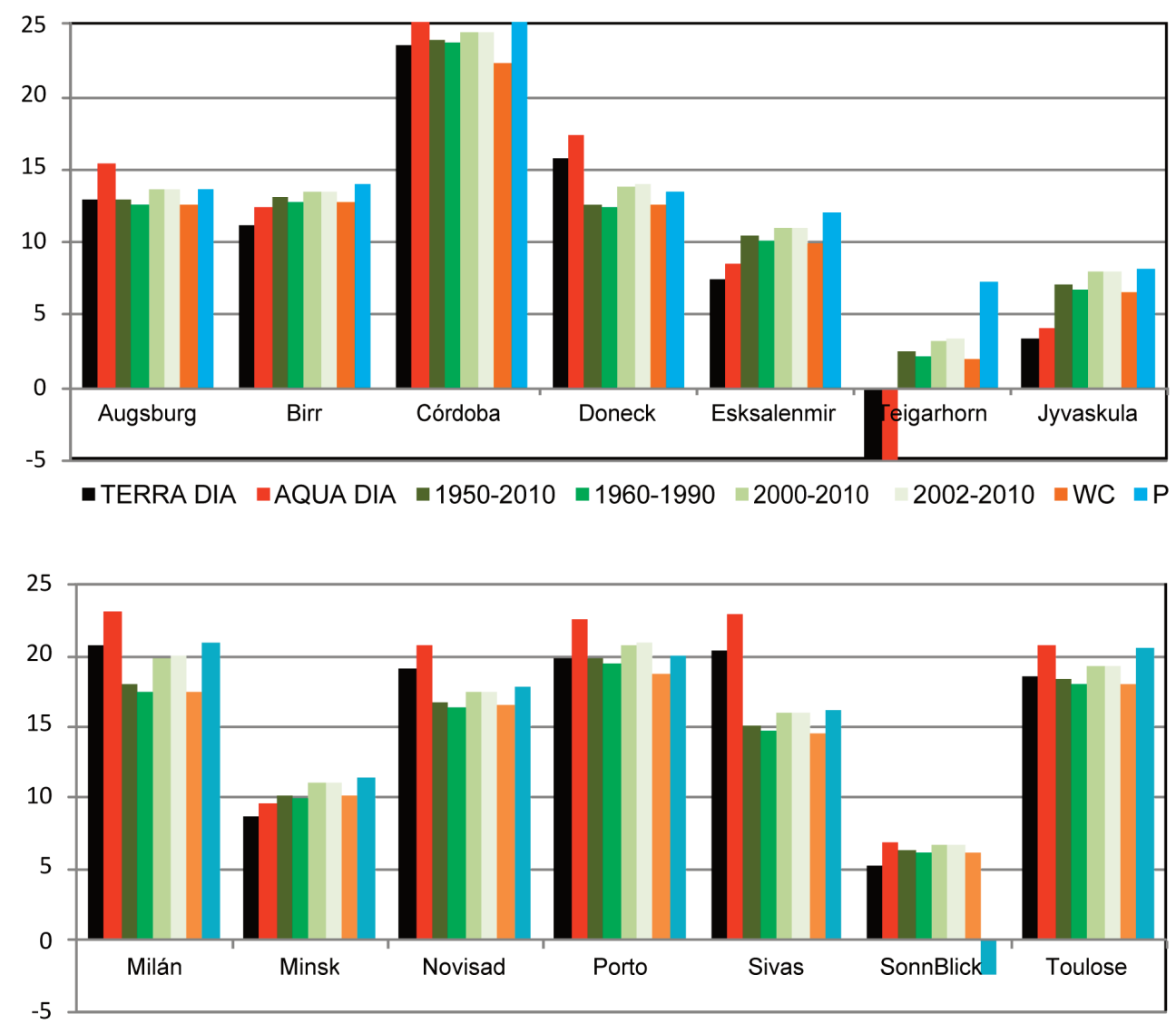

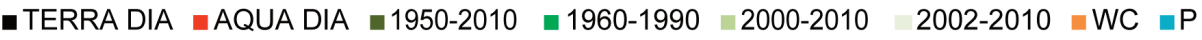

Figura 12. Datos de TMax para cada fuente de datos en cada estación de control (las fechas indican rangos de años de la información sacada de la base de datos ECA\&D, WC son las temperaturas de WorldClim y P es el valor real puntual de la estación). 


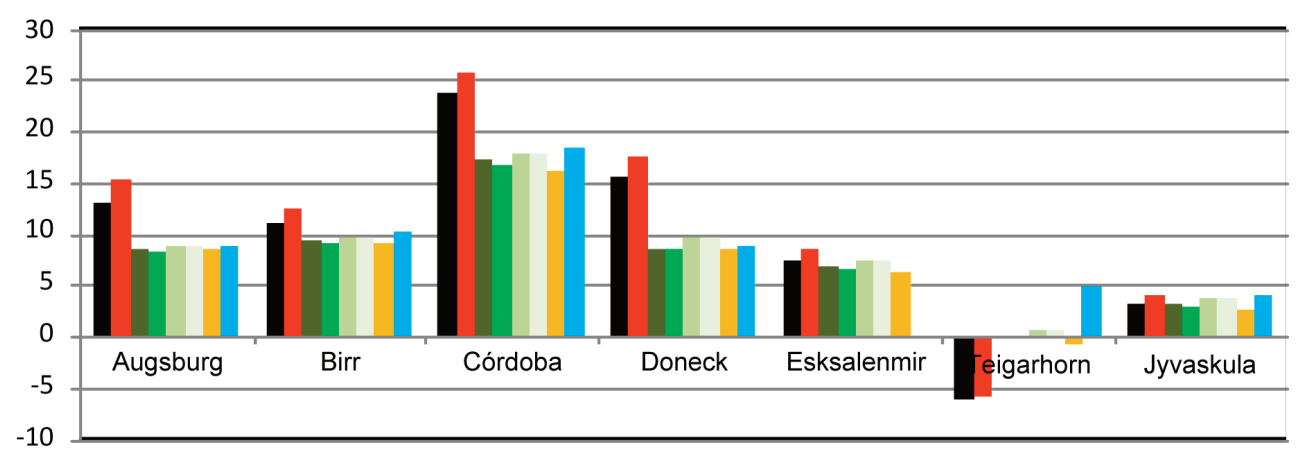

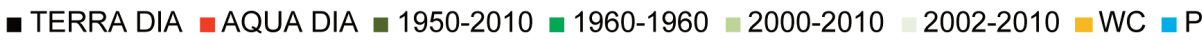

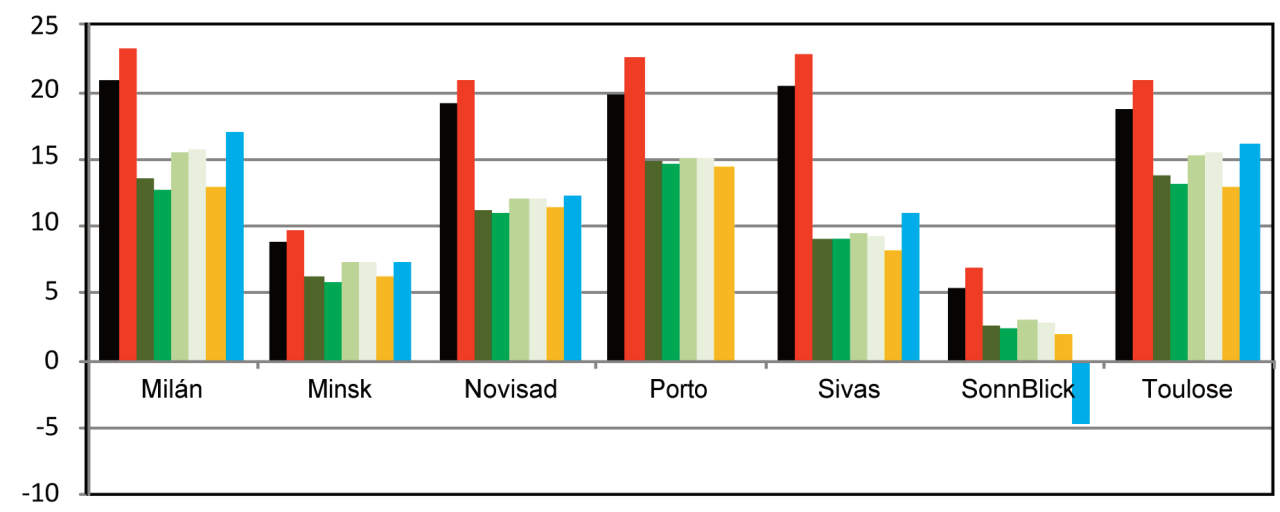

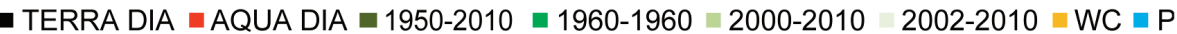

Figura 13. Datos de TMedia para cada fuente de datos en cada estación de control (las fechas indican rangos de años de la información sacada de la base de datos ECA\&D,

WC son las temperaturas de WorldClim y P es el valor real puntual de la estación).

\subsubsection{Análisis de la temperatura mínima (TMin) por estaciones}

La Figura 14 (Tablas 2 y 3) constata que existen problemas en las tres bases de datos utilizadas a la hora de modelizar correctamente los datos de TMin en la zona de Islandia y de los Alpes. En el resto de estaciones de control, las diferencias con respecto al valor real son inferiores a los $4^{\circ} \mathrm{C}$. Un análisis en profundidad permite observar como MODIS Terra ajusta mejor que Aqua con el dato real de ECA\&D en la totalidad de las estaciones, mientras que WorldClim muestra un error semejante en todas 


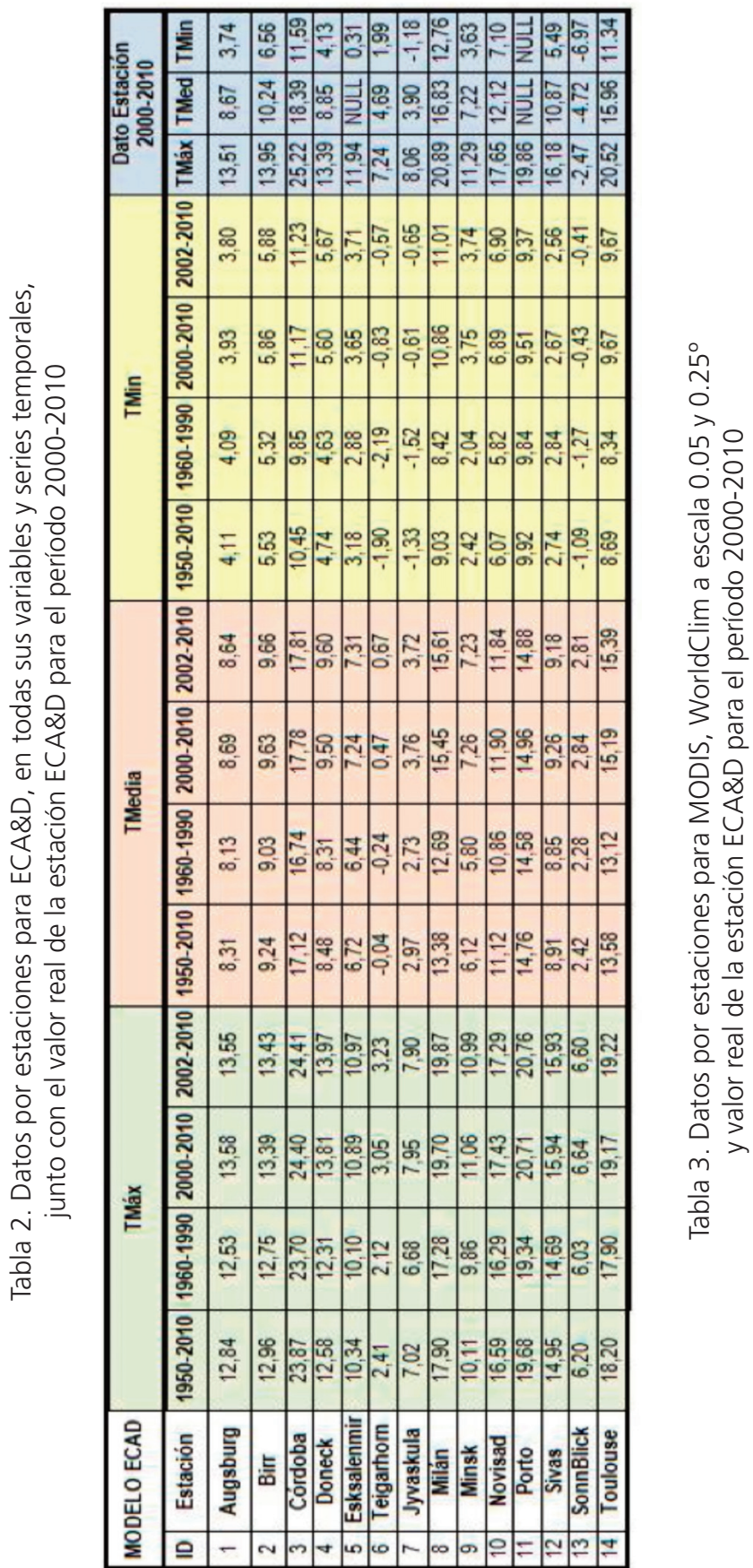

\begin{tabular}{|c|c|c|c|c|c|c|c|c|c|c|c|c|c|c|c|}
\hline \multirow{3}{*}{ 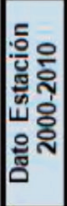 } & $\underset{\sum}{\underline{\Sigma}}$ & $\begin{array}{l}\text { 足 } \\
\text { m. }\end{array}$ & $\mid \begin{array}{l}\mathscr{B} \\
0 \\
0\end{array}$ & 象 & $\frac{m}{\sigma}$ & m. & $\frac{8}{-}$ & $\frac{\infty}{7}$ & & & & & 㝵 & & 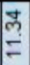 \\
\hline & 変 & 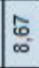 & 䓌 & 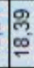 & 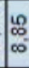 & $\frac{1}{2}$ & $\begin{array}{l}8 \\
0\end{array}$ & 8. & 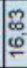 & I & $\begin{array}{l}\frac{N}{N} \\
- \\
\end{array}$ & & 合 & & 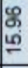 \\
\hline & 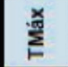 & $\begin{array}{l}\bar{\omega} \\
\stackrel{5}{\sigma}\end{array}$ & $\stackrel{2}{2}$ & సี & శ్ & 鹿 & $\stackrel{d}{N}$ & 8 & 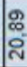 & $\stackrel{\text { జి }}{=}$ & 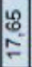 & 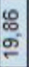 & \begin{tabular}{l}
$\infty$ \\
\hdashline \\
0 \\
\hdashline
\end{tabular} & 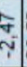 & 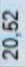 \\
\hline \multirow{6}{*}{ 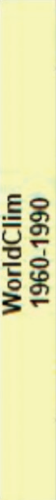 } & 듵 웅 & $\stackrel{8}{\circ}$ & 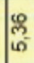 & \% & & $\stackrel{\infty}{\infty}$ & F & مُ & జิ & $\%$ & $\begin{array}{c}\bar{y} \\
\bar{c} \\
0\end{array}$ & 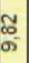 & $\stackrel{p}{=}$ & & 离 \\
\hline & $\sum_{k}^{E}$ & $\frac{\infty}{\sigma}$ & $\underset{\mathbb{N}}{\mathbb{N}}$ & \% & लु & $\bar{i}$ & 角 & $\overline{\underline{N}}$ & $\underset{\infty}{9}$ & $\frac{\infty}{i}$ & $\mid \begin{array}{l}5 \\
0 \\
0\end{array}$ & \begin{tabular}{l}
$\hat{n}$ \\
\hdashline \\
0
\end{tabular} & $\because$ & & 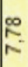 \\
\hline & 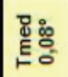 & $\underset{\substack{2 \\
\infty \\
\infty}}{\infty}$ & $\bar{\sigma}$ & 㞭 & & $\dddot{m}$ & 永 & 8 & $\%$ & 8 & $\begin{array}{l}- \\
= \\
=\end{array}$ & $\frac{\infty}{2}$ & 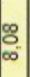 & $\frac{8}{0}$ & $\begin{array}{l}\mathscr{2} \\
\stackrel{2}{\underline{N}}\end{array}$ \\
\hline & Е્屯 & 應 & ळ్ & $\begin{array}{l}8 \\
0\end{array}$ & œ & $\frac{\infty}{\infty}$ & {$\left[\begin{array}{l}\infty \\
\infty \\
0 \\
\hdashline\end{array}\right.$} & 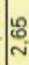 & צ & 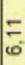 & $\begin{array}{l}\mathbb{N} \\
= \\
=\end{array}$ & \begin{tabular}{l}
$\widehat{m}$ \\
\multirow{2}{0}{}
\end{tabular} & 8 & 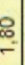 & I \\
\hline & 商。 & 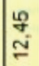 & $\begin{array}{l}\bar{S} \\
\text { ㄸ }\end{array}$ & $\begin{array}{l}8 \\
\text { in }\end{array}$ & & 8 & م. & 赵 & J & $\mathscr{~}$ & $\begin{array}{l}0 \\
0 \\
0 \\
0 \\
0\end{array}$ & $\begin{array}{l}8 \\
0 \\
0 \\
0 \\
0\end{array}$ & \begin{tabular}{l}
$\bar{n}$ \\
\pm \\
\hdashline
\end{tabular} & & 占 \\
\hline & 欷 & 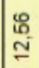 & 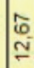 & $\tilde{A}$ & I & 2. & 8 & $\begin{array}{l}\mathscr{0} \\
0 \\
0\end{array}$ & స్ & 웅 & $\left|\begin{array}{|c}0 \\
\vdots \\
0 \\
0 \\
0\end{array}\right|$ & \begin{tabular}{|l}
$\overline{0}$ \\
$\infty$ \\
0
\end{tabular} & $\begin{array}{l}\text { y } \\
\text { y }\end{array}$ & $\frac{\bar{\sigma}}{\omega}$ & 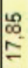 \\
\hline \multirow{8}{*}{ 을 } & 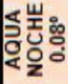 & N & $\stackrel{\mathscr{N}}{\mathbb{N}}$ & 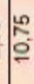 & & $\frac{g}{2}$ & & $\begin{array}{l}18 \\
7\end{array}$ & & 8 & $\left|\begin{array}{c}\infty \\
\vdots \\
+\end{array}\right|$ & $\begin{array}{l}\text { ప } \\
\vdots \\
0 \\
0\end{array}$ & $\frac{\pi}{0}$ & & $\frac{O}{N}$ \\
\hline & 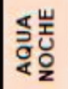 & 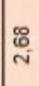 & $\stackrel{8}{\mathrm{~m}}$ & 흥 & 禺 & $\ddot{0}$ & & గ్ & $\bar{m}$ & $\begin{array}{l}8 \\
0 \\
0\end{array}$ & $\left.\mid \begin{array}{l}n \\
0 \\
\sigma^{2}\end{array}\right]$ & $\frac{7}{\sigma}$ & $\frac{7}{7}$ & & 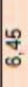 \\
\hline & 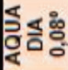 & $\begin{array}{l}0 \\
\pm \\
\end{array}$ & $\widehat{\widehat{c}}$ & 离 & 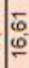 & $\mathbb{m}_{\infty}$ & $\begin{array}{l}0 \\
0 \\
0 \\
\end{array}$ & $\begin{array}{l}8 \\
0\end{array}$ & స్ & $\stackrel{8}{=}$ & $\frac{\mathscr{m}}{\bar{N}}$ & $\begin{array}{l}\text { in } \\
\vdots \\
0\end{array}$ & & $\frac{0}{6}$ & $\frac{8}{\pi}$ \\
\hline & 造文 & స్ & $\stackrel{m}{\stackrel{n}{7}}$ & 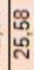 & $\stackrel{m}{=}$ & $\underset{\infty}{ }$ & $\begin{array}{l}\% \\
\% \\
10\end{array}$ & \% & ฮิ & के & 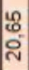 & 过 & 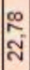 & & 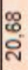 \\
\hline & 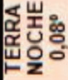 & $\overline{0}$ & Fे & 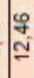 & & & & $\stackrel{E}{-}$ & & & \% & 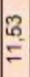 & సิ & & लि \\
\hline & 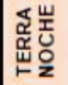 & $\bar{\tau}$ & $\ddot{B}$ & $\stackrel{8}{\circ}$ & & & & $\frac{\infty}{\div}$ & & & 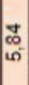 & 芯 & 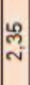 & & $\frac{2}{\pi}$ \\
\hline & 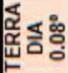 & 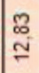 & 点 & 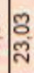 & 5 & $\overline{-}$ & $\frac{m}{-}$ & న్ & ஸึ & ळ & $\begin{array}{l}\dddot{8} \\
0 \\
\sigma\end{array}$ & $\begin{array}{l}\mathscr{2} \\
\stackrel{\sim}{2} \\
\stackrel{2}{2}\end{array}$ & & $\begin{array}{c} \pm \\
m \\
m\end{array}$ & $\begin{array}{l}\mathscr{f} \\
\stackrel{乛}{-}\end{array}$ \\
\hline & 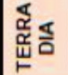 & $\stackrel{\Xi}{\stackrel{\Xi}{\simeq}}$ & $\stackrel{\circ}{=}$ & 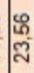 & 5 & ని & $\begin{array}{l}\bar{y} \\
\varphi \\
\varphi\end{array}$ & $\bar{N}$ & ঊ & $\sim$ & \% & $\begin{array}{l}k \\
\sigma \\
\sigma\end{array}$ & & $\begin{array}{c}\overline{\tilde{n}} \\
\bar{\omega}\end{array}$ & 艿 \\
\hline & 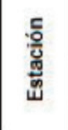 & 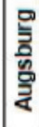 & 흐 & 응 & 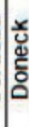 & 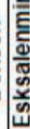 & $\begin{array}{l}\frac{5}{0} \\
\frac{2}{0} \\
\frac{0}{2}\end{array}$ & $\frac{2}{3}$ & 告 & 5 & 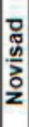 & 응 & 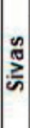 & & 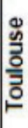 \\
\hline & 은 & - & $\sim$ & $m$ & o & (1) & 10 & $1^{12}$ & 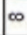 & Se & $F$ & $=$ & $\cong$ & $m$ & \pm \\
\hline
\end{tabular}



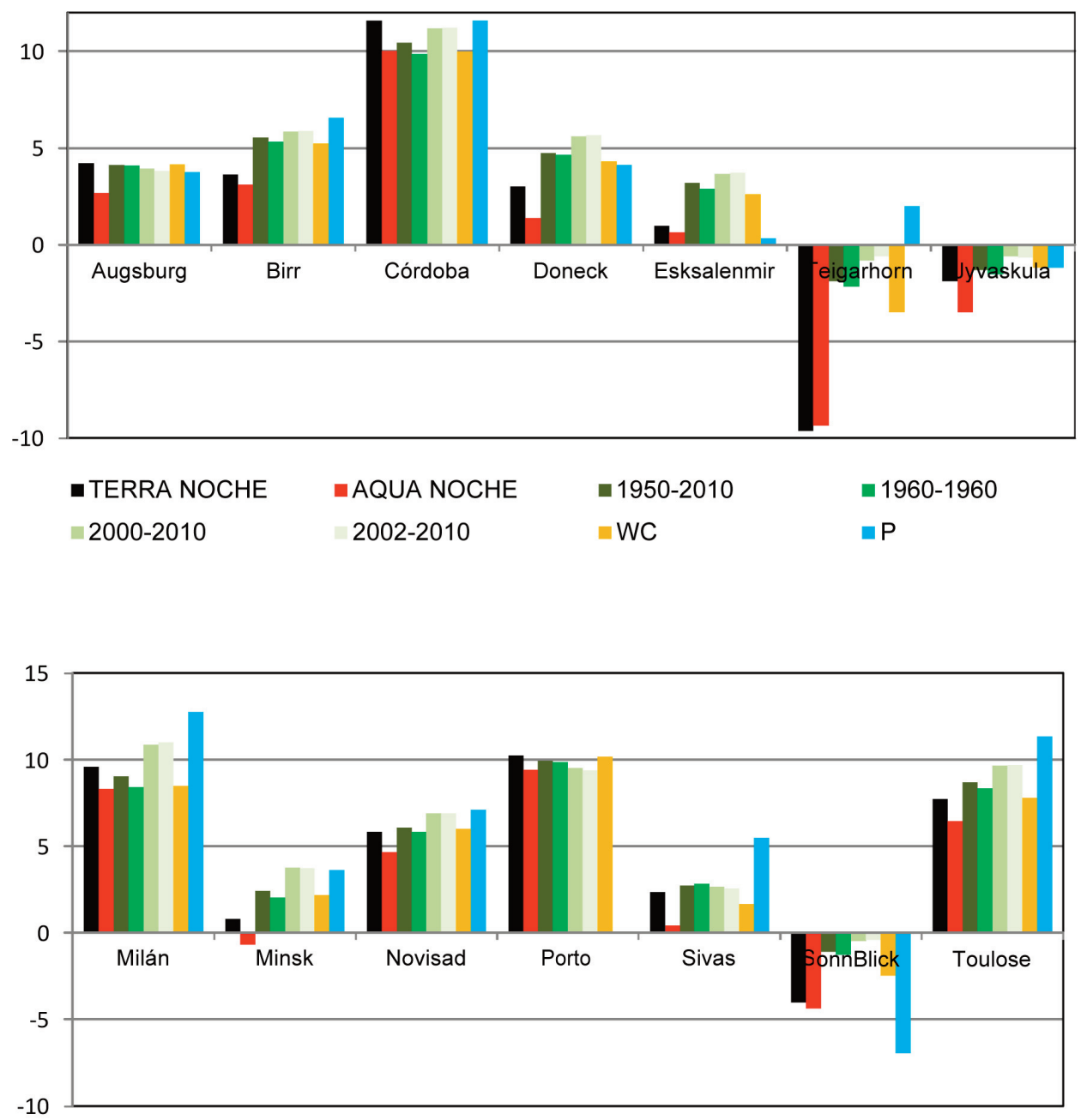

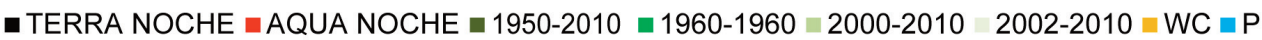

Figura 14. Datos de TMin para cada fuente de datos en cada estación de control (las fechas indican rangos de años de la información sacada de la base de datos ECA\&D, WC son las temperaturas de WorldClim y P es el valor real puntual de la estación). 
las estaciones del mediterráneo mostrando un mejor ajuste en las estaciones de clima continental y las del Este de Europa. Finalmente, la base de datos ECA\&D muestra una alta coincidencia en todas las estaciones con respecto al valor puntual, con la excepción ya señalada de aquellas situadas en terrenos fríos y generalmente helados.

\subsection{Análisis por climas LandMap2 level 1}

\subsubsection{Análisis de la temperatura máxima (TMax)}

Como se observa en la Figura 15, los datos MODIS presentan las mayores diferencias positivas con respecto a WorldClim y ECA\&D en los climas del sur de Europa, es decir, en el Mediterráneo, en el Estepario y en el Turco-Anatolia, mientras que las diferencias negativas se dan en los climas Boreal y Alpino, es decir, en los más fríos. En el clima Atlántico y en el Continental, las diferencias son escasas. En lo que respecta a las diferencias entre la información suministradas por los dos satélites MODIS, se observa que Aqua recoge datos de temperatura máxima media superiores a Terra.

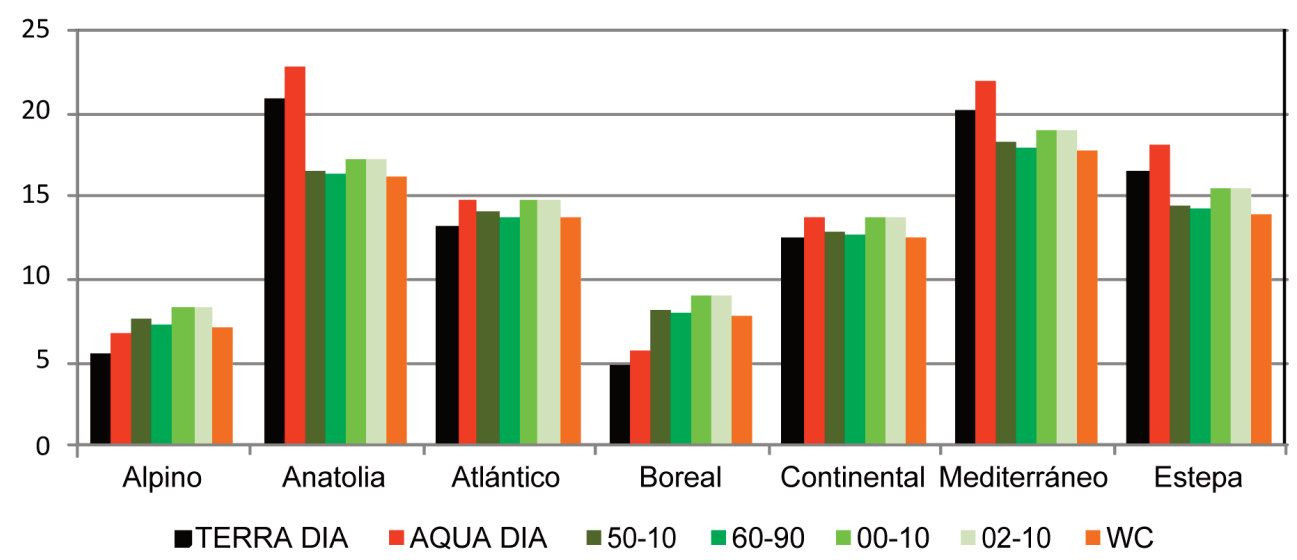

Figura 15. Datos de TMax por climas Landmap2 y fuente de datos.

En cuanto a la comparación entre las bases de datos que utilizan información extraída de estaciones meteorológicas, señalar que WorldClim y ECA\&D presentan una gran similitud, siendo los datos 1960-1990 muy semejantes entre sí (Tablas 4 y 5). Por último señalar que los datos de ECA\&D presentan temperaturas más altas cuanto más recientes son los rangos de años considerados. 

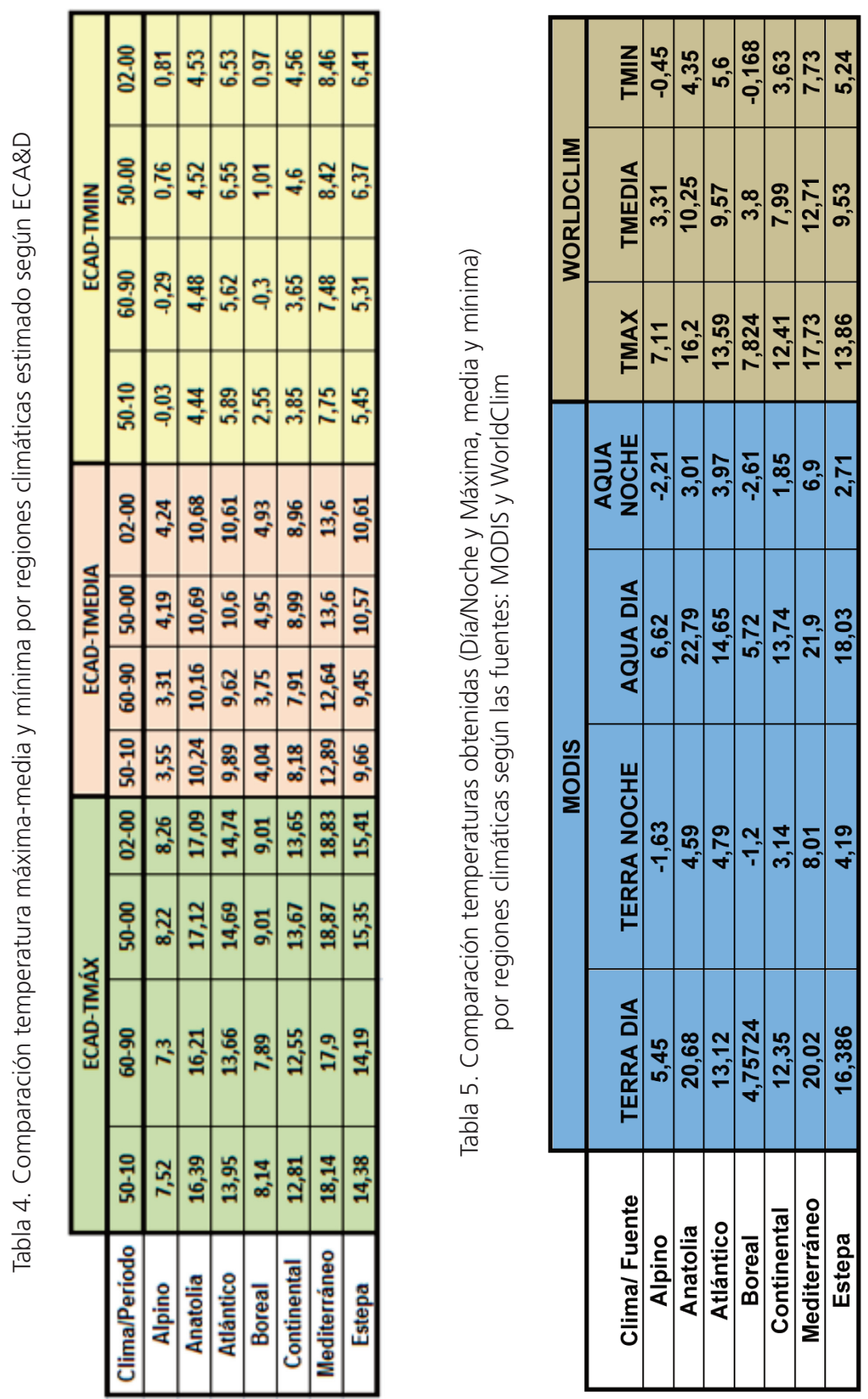


\subsubsection{Análisis de la temperatura media (TMedia)}

El análisis de esta variable por climas muestra que existen grandes diferencias entre los datos que aporta MODIS con los del valor real y con los de las series de datos procedentes de estaciones meteorológicas (Figura 16 y Tablas 4 y 5), siendo en este caso los valores ofrecidos por Aqua siempre superiores a los de Terra.

Las mayores diferencias entre las series de WorldClim y ECA\&D se reflejan en el clima Alpino y Boreal, observándose temperaturas más altas en las series del siglo XXI respecto a los datos más antiguos.

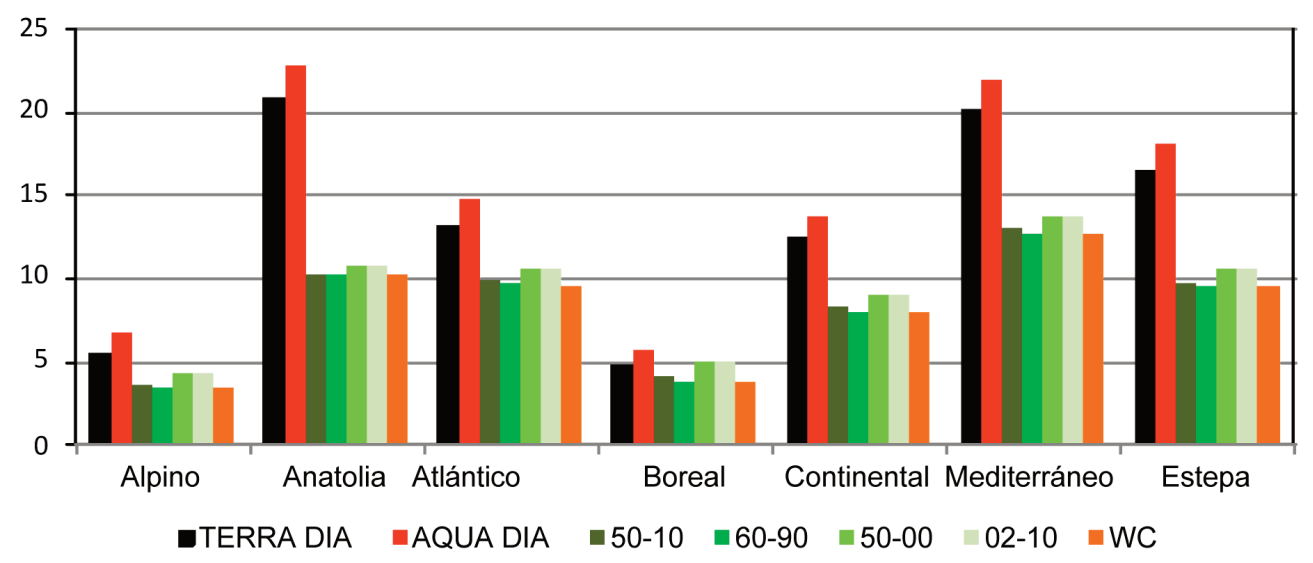

Figura 16. Datos de TMedia por climas Landmap2 y fuente de datos.

\subsubsection{Análisis de la temperatura mínima (TMin)}

La Figura 17 (Tablas 4 y 5) muestra que existen escasas diferencias entre las fuentes de datos salvo en el caso del clima Boreal. En esta variable, MODIS Aqua es la fuente de datos que en todos los casos presenta datos más alejados de la tendencia común marcada por el resto. Su satélite gemelo, MODIS Terra, se ajusta bien con el resto de fuentes en todos los climas salvo Alpino y Boreal.

Los datos de ECA\&D son para el mismo periodo siempre superiores a WorldClim y a los de MODIS salvo en el caso de los climas del Sur de Europa, donde se igualan. 


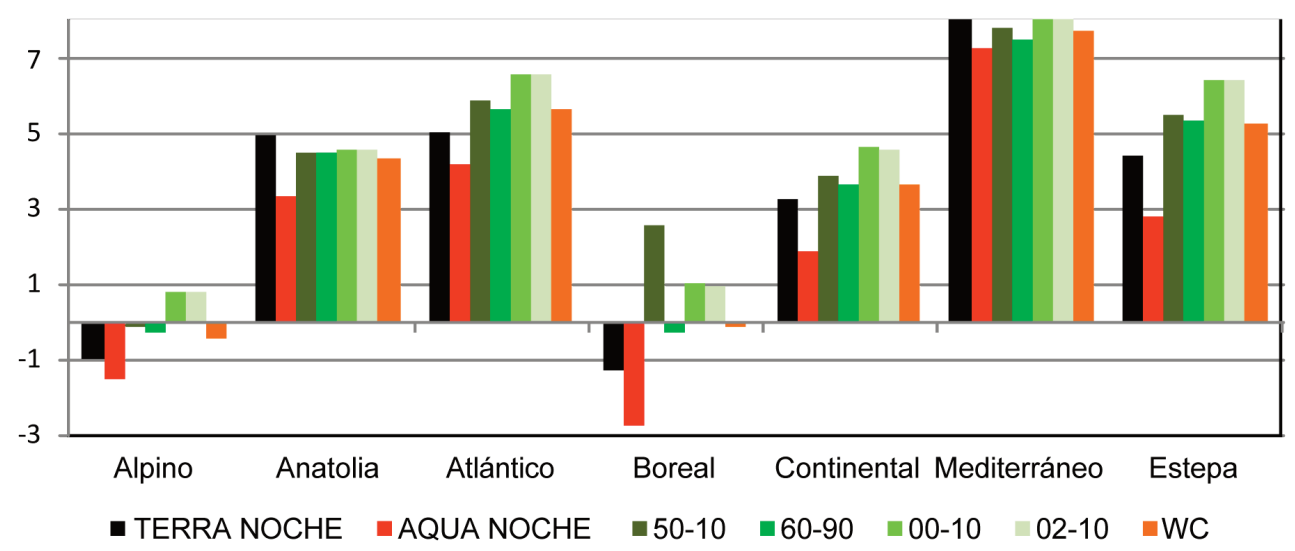

Figura 17. Datos de TMin por climas Landmap2 y fuente de datos.

\section{Discusión}

El análisis estadístico y las distintas escalas espaciales y temporales utilizadas en este trabajo muestran que en el territorio europeo no existen diferencias estadísticamente significativas entre ellas, mostrando un limitado rango de error entre $+/-3 \mathrm{y}+/-5{ }^{\circ} \mathrm{C}$ entre las tres bases de datos utilizadas para conocer la TMax, la TMedia y la TMin. Dichos resultados son coherentes con la veracidad científica de estas fuentes, ya que han sido creadas, testadas y puestas a disposición de la comunidad científica por parte de entidades de reconocido prestigio, constituyendo en la actualidad algunas de las series más frecuentemente utilizadas en el desarrollo de cualquier estudio de ciencias experimentales a este tipo de escalas medianas y pequeñas (Zaksek et al., 2009).

Es necesario destacar que se han recogido dos variables distintas temperatura: temperatura de superficie y temperatura del aire ( $2 \mathrm{~m}$ sobre el nivel del suelo) (Mildrexler et al., 2011). De esta forma, se comparan dos procedimientos de captura de datos y, además, distintas formas de procesar la información base. Se decidió incluir ambas variables dado que su libre disposición, facilidad de acceso y su contrastada calidad científica hace que hayan sido usados de manera masiva en diversos estudios de ciencias experimentales en los que disponer de un dato climático es necesario para proyectar un modelo. Es por ello que se comparan datos espacialmente o temporalmente no coincidentes (Emamifar et al., 2013; Peón et al., 2014; Janatian et al., 2017). Sin embargo, es necesario señalar que queda fuera de este estudio todo proceso técnico de optimización y corrección de datos para la integración de ambas variables de temperatura.

Aunque las variables estén correlacionadas y las series de temperatura sean científicamente validas, conocer sus limitaciones y diferencias es vital, dado que en el mo- 
delado de cualquier proceso ambiental, un pequeño error introduce un alto grado de incertidumbre en los resultados finales de estos análisis. Así, diferencias de un grado en la monitorización diaria de la temperatura para una periocidad anual para, por ejemplo, estimar la posibilidad de presencia de una especie, la eclosión de un huevo, de riesgo de helada o de la estimación de consumo energético por aire acondicionado / calefacción, alcanzaría una variación de $365^{\circ} \mathrm{C}$, lo que provocaría alteraciones importantes en el área afectada, en los días estimados, los costes de intervención, etc. calculados (Gray et al., 2008; Benito et al., 2011; Campbell-Lendrum et al., 2015).

La comparación de las bases WorldClim y ECA\&D constata que existe una gran semejanza entre los datos de temperatura que proporcionan. Esta situación era esperada dado que ambas comparten en gran parte las mismas fuentes para la construcción de sus modelos. Pese a ello, se comprueba en el análisis puntual que para las misma estaciones de

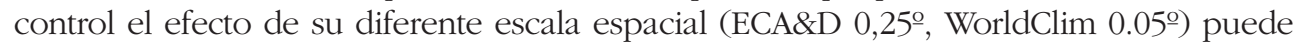
provocar diferencias de entre $0,3^{\circ} \mathrm{C}$ y $7^{\circ} \mathrm{C}$. De igual forma, a escala de regiones climáticas se comprueba una mayor suavidad de los datos de WorldClim respecto a ECA\&D. Esta circunstancia puede deberse a la mayor correlación espacial que existe en WorldClim con respecto a ECA\&D, dado que dispone de una red inferior de estaciones de referencia que esta última para el desarrollo de su modelo como a su escala original de trabajo.

Las diferencias más grandes se comprueban en zonas abruptas y/o con una gran variabilidad térmica, siendo su concordancia muy pareja en las zonas más llanas (por ej. grandes llanos y mesetas europeas) y con climas térmicamente más homogéneos (latitudes medias). Asimismo, se constata una de las limitaciones propias de todos los modelos basados a partir de puntos de referencia: la falta de una densidad de puntos para recoger toda la heterogeneidad existente de la temperatura en estas zonas, ya sea por efecto del relieve en zonas montañosas, por la escasez de puntos (zonas desérticas o no pobladas) o la mayor variabilidad térmica de estos territorios. Esta limitación no logra ser superada por ECA\&D, a pesar de que esta base de datos dispone de más de 20.000 puntos para la zona de estudio, dado que refleja las misma debilidades que WorldClim (Hofstra et al., 2010; Deblauwe et al., 2016).

Junto a este problema, otros dos fenómenos que deben ser considerados de cara a tener en cuenta el uso de este tipo de bases de datos, son la auto-correlación y la falta de homogeneidad en la recogida de datos de las estaciones meteorológicas (Hofstra et al., 2009; van den Besselaar et al., 2011; Estrada-Peña et al., 2014). De esta forma y, a tenor de los resultados obtenidos, podemos considerar que a la escala de trabajo utilizada, estos modelos son útiles en latitudes medias y climas templados en zonas con una alta densidad de puntos de validación.

Independientemente de las diferencias señaladas, todas las series temporales de datos ECA\&D reflejan un gradiente positivo de la temperatura cuanto más reciente es la escala temporal de análisis, lo que está de acuerdo con el proceso de cambio climático vigente (van der Schrier et al., 2013). Los datos históricos de WorldClim solo recogen 
los datos de 1960-1990 lo que, teniendo en cuenta el incremento señalado, limita su utilidad. De igual forma, su menor disponibilidad de puntos y su desigual distribución limita su uso respecto a la creciente funcionalidad de ECA\&D para Europa, dada su actualización y progresiva densificación de más puntos de control. En todo caso, queda constatada la limitación intrínseca de todos los modelos basados en puntos señalada anteriormente de requerir un gran número de estaciones sobre el territorio distribuidas regularmente sobre este para obtener unos buenos resultados a escalas medias de trabajo, siendo la demanda de estaciones superior cuánto más variable sea el territorio o la dinámica de la temperatura. Esto es un problema logístico difícil de solucionar en muchos casos. Para hacerlo se requerirá de un complejo post-procesado estadístico inexacto (Upscaling, downscaling...) para su ajuste a otras escalas. (Moreno et al., 2016).

Centrándonos en los datos proporcionados por MODIS, los resultados muestran que éstos ajustan en un rango de $2^{\circ} \mathrm{C}$ para la fase diurna y $5^{\circ} \mathrm{C}$ para la fase nocturna, siendo el ajuste casi exacto en latitudes medias europeas. Así, muestra coincidencia en cuanto a las zonas de debilidad en el modelado con las bases de datos ECA\&D y WorldClim, aunque se deben a motivos distintos. En este sentido, el equipo científico de MODIS reconoce limitaciones en las regiones próximas a los polos debido a la órbita polar de este satélite y en entornos climáticos extremos (fríos y cálidos) debido a su sensor (Wan, 2007). A esto hay que añadir otros problemas como la presencia de nubes no detectadas (Westermann et al., 2011) y la presencia de superficies heterogéneas dentro del pixel (Klene et al., 2011). Dichas limitaciones están siendo investigadas y mejoradas de forma continua por parte del citado equipo mediante la aplicación de nuevos algoritmos.

Los resultados obtenidos señalan que MODIS no es una fuente válida en el territorio europeo para utilizarlo como dato de TMedia del aire, dado que presenta importantes diferencias respecto de estos, ya que temporalmente MODIS recoge 4 datos puntuales a lo largo del día de la temperatura de superficie de forma que es difícil que estos datos puedan ajustar con el valor promedio recogido por las estaciones meteorológicas a lo largo de un día. Además, como ha sido resaltado previamente, el dato de temperatura que recoge es diferente al de la temperatura del aire.

Del análisis de los datos se observa que el uso de valores medios tanto de la TMax como de la TMin parece justificar el mejor ajuste de Terra con respecto al de Aqua. En este sentido, la hora de paso de MODIS por el territorio europeo es fundamental para explicar estas diferencias. Así, tal y como se señala en Alonso et al. (2013), el desajuste entre Terra y Aqua en la hora de sus capturas provoca la variabilidad en los resultados respecto de los de temperatura del aire dada sus diferencias cualitativas y temporales (esta no se tiene porque ajustar a la dinámica de los distintos climas presentes en el territorio europeo). De esta manera, aun suponiendo un algoritmo perfectamente calibrado para obtener la temperatura de superficie de forma exacta con las imágenes MODIS, recoger al mismo tiempo el dato de temperatura Máxima a las $10.30 \mathrm{~h}$ (Terra) o 13.30 (Aqua) en una zona Boreal o en una Estepa Mediterránea implica una aproximación a la realidad de estos climas que debe valorarse de cara a su uso en el estu- 
dio de cualquier proceso ambiental, inclusive para con correlación con la temperatura del aire. Aun así, hay que reseñar que los mejores ajustes con MODIS se consiguen para TMax y TMin con Terra, con la única excepción de la las zonas con clima Alpino y el territorio de Islandia). En el caso de Aqua, la TMax es la variable que más variabilidad recoge sobrepasando el rango real con mayor amplitud. En el caso de TMin recoge el rango térmico con valores más bajos. En ambos casos su método de captura parece justificar dichos resultados y variabilidad.

De esta manera, los datos de temperatura de superficie ofrecidos por MODIS, especialmente Terra, ofrecen una concordancia respecto a la temperatura del aire máxima y mínima semejante a las propias series ECA\&D y WorldClim que recogen este dato. La distinta resolución espacial entre las series hizo necesario homogeneizar los datos hasta la mayor escala de estas $\left(0,25^{\circ}-0,05\right)$. Es importante destacar por tanto de cara a su utilidad que MODIS ofrece de datos científicamente validados con una resolución 5 veces mayor (Wan, 2004; Wan et al., 2008) a los mapas desarrollados a partir de las estaciones meteorológicas abarcando gracias a ello un rango espacial que estos modelos no pueden igualar a escala global. Así, tal y como indica Neteler (2010) ofrece un dato que, cuanto menos, sirve para modelar la variable temperatura en zonas con escasez de estaciones o distribución irregular.

Por último, señalar la posibilidad que ofrece MODIS de disponer de cuatro medidas diarias de temperatura de superficie, abriendo la posibilidad de disponer de unos datos intradiarios que amplían las posibilidades de análisis con respecto de los datos diarios estandarizados de ECA\&D, pudiendo a su vez correlacionar ambas variables térmicas al menos respecto a TMax y TMin. Esta posibilidad se vería aún más ampliada en caso de utilizarse alguno de sus productos a escalas de trabajo espaciales mayores (por ejemplo, $1 \mathrm{~km}^{2}$ ). En este contexto, los productos MODIS de temperatura de superficie presentan un mayor abanico de posibilidades para su utilización en el estudio de procesos ambientales a escalas medias y grandes con un alto grado de exactitud que las bases de datos WorldClim y ECA\&D (Van Doninck et al., 2014).

\section{Conclusión}

Entidades como la NASA, la Unión Europea, la ONU y universidades de todo el mundo están obteniendo de forma continua una gran cantidad de datos de distintas variables de interés que se publican de manera gratuita mediante la utilización de bases de datos OpenSource. Este fenómeno, junto al avance de la tecnología que permite explotarlos (principalmente herramientas estadísticas y SIG) ha facilitado y multiplicado la realización de estudios científicos de distinta índole. Sin embargo, la facilidad para la accesibilidad a los datos no debe hacer olvidar que la correcta selección de las fuentes de datos a utilizar en relación con los objetivos e hipótesis planteadas es un proceso básico para el correcto desarrollo de un trabajo científico. 
En este trabajo se ha analizado la variable temperatura desde una índole climática como variable recurrente para el análisis de innumerables procesos socio-ambientales recurriendo para ello a series validadas y de uso científico habitual de dos variables térmicas diferentes que capturan y procesan sus datos en dos procesos totalmente distintos. Se ha comprobado la homogeneidad y veracidad científica del dato temperatura como valor climático (entendido como valor promedio del conjunto de la serie) en todas estas series pese a la diferencia temporal y cualitativa entre ellas.

Se destaca con ello la figura del usuario experto, que debe seguir acercándose a ellas con el conocimiento de sus limitaciones para generar, tratar o utilizar estos datos con coherencia y de acuerdo al uso óptimo requerido en cada ocasión para poder obtener el resultado veraz.

Junto a ello, se constatan las diferencias cualitativas y temporales entre las variables como ya se han descrito a lo largo de todo el estudio que deben ser tenidas en cuenta al analizar las mismas. Así, se ha constatado que a nivel europeo y considerando una escala de trabajo media $\left(0.25^{\circ}\right)$, la base de datos de temperatura ECA\&D, gracias a su mayor número de estaciones y continuidad en el tiempo, mejora en prestaciones a Worldclim. La principal debilidad de esta base de datos reside en las zonas con un clima frio extremo (Islandia y zona de los Alpes), así como su escala espacial. WorldClim quedaría limitado para usos más generalistas dentro de su período de datos (1960-1990). Respecto a esta última, señalar que en la actualidad se está creando una nueva versión, la 2.0, que ofrecerá datos para el periodo 1970-2000.

En cuanto a la información de temperatura aportada por MODIS en el área de estudio, se constata que ofrecen un buen ajuste entre ambas variables para TMax y TMin principalmente a través de Terra, no siendo optima su utilización para la TMedia del aire al ser variables cualitativamente distintas cuyo método de captura impiden estimar adecuadamente su resultado. De esta forma, MODIS y el resto de fuentes procedentes de la teledetección, gracias a su gran variabilidad temporal y espacial, se perfilan como una buena fuente de datos para TMax y TMin en caso de necesidad de escalas mayores que, en estos momento, ECA\&D y WorldClim no ofrecen (Wan.Z 2007; Neteler et al., 2014).

Por último, hay que destacar los avances que se están haciendo en la integración de las variable temperatura del aíre y temperatura de superficie para un óptimo filtrado y calibrado de modelos térmicos-climáticos (Sterwart et al., 2016). No obstante, es necesario señalar que MODIS presenta incertidumbres en zonas de condiciones extremas y latitudes entorno al Círculo Polar que hay que tener en cuenta. De esta manera, se debe proseguir con el esfuerzo de conseguir algoritmos o sensores más precisos que minimicen los errores de estimación observados. 


\section{Bibliografía}

Alonso-Carné, J., García-Martín, A., Estrada Peña, A., 2013. Systematic errors in temperature estimates from MODIS data covering the western Palearctic and their impact on a parasite development model. Geospatial Health 8(1), 2013, pp. 1-12.

Benito, B., Lorite, J., Peñas, J., 2011. Simulating potential effects of climatic warming on altitudinal patterns of key species in Mediterranean-alpine ecosystems. Climatic Change, 108(3), pp. 471-483.

Bustamante, J., 2003. Cartografía predictiva de variables climáticas: comparación de distintos modelos de interpolación de la temperatura en España peninsular. Graelsia 59 (2-3), pp. 359-376.

Campbell-Lendrum, D., Manga, L., Bagayoko, M., Sommerfeld, J., 2015. Climate change and vector-borne diseases: what are the implications for public health research and policy? Phil. Trans. R. Soc. B 370: 20130552.

Dantas-Torres, F., 2015. Climate change, biodiversity, ticks and tick-borne diseases: The butterfly effect. International Journal for Parasitology: Parasites and Wildlife, 4(3), pp. 452-461.

Deblauwe, V., Droissart, V., Bose, R., Sonké, B., Blach-Overgaard, A., Svenning, J.-C., Wieringa, J.J., Ramesh, B.R., Stévart, T. and Couvreur, T.L.P., 2016. Remotely sensed temperature and precipitation data improve species distribution modelling in the tropics. Global Ecology and Biogeography, 25, pp. 443-454.

ECA\&D Project Team \& Royal Netherlands Meteorological Institute KNMI, European Climate Assessment \& Dataset (ECA\&D) disponible en: http://www.ecad.eu/documents/atbd.pdf

Emamifar, S., Rahimikhoob, A. and Noroozi, A.A., 2013, Daily mean air temperature estimation from MODIS land surface temperature products based on M5 model tree. Int. J. Climatol., 33: pp. 3174-3181. doi:10.1002/joc.3655.

Estrada-Peña, A., Estrada-Sánchez, A., Estrada-Sánchez, D., de la Fuente, J., 2013. Assessing the effects of variables and background selection on the capture of the tick climate niche. Int J Health Geogr., 12:43.

Field, C.B. (Ed.). 2012. Managing the risks of extreme events and disasters to advance climate change adaptation: special report of the intergovernmental panel on climate change. Cambridge University Press.

Fordham, D.A., Akçakaya, H.R., Araújo, M.B., Keith, D.A., Brook, B.W., 2013. Tools for integrating range change, extinction risk and climate change information into conservation management. Ecography, 36, pp. 956-964.

Geiger R., 1965. The climate near the ground. Mass, Cambridge, 611.

Gray, J.S., Dautel, H., Estrada-Peña, A., Kahl, O., Lindgren, E., 2009. Effects of Climate Change on Ticks and Tick-Borne Diseases in Europe, Interdisciplinary Perspectives on Infectious Diseases, vol. 2009, Article ID 593232, 12 pages.

Haylock, M.R., Hofstra, N., Klein Tank, A.M.G., Klok, E.J., Jones, P.D., New, M., 2008. A European daily high-resolution gridded data set of surface temperature and precipitation for 19502006, J. Geophys. Res., 113, D20119. 
Hijmans, R.J., Cameron, S.E., Parra, J.L., Jones, P.G., Jarvis, A., 2005. Very high resolution interpolated climate surfaces for global land areas. International journal of climatology, 25(15), 1965-1978.

Hofstra, N., Haylock, M., New, M., Jones, P.D., 2009. Testing E-OBS European high-resolution gridded data set of daily precipitation and surface temperature, J. Geophys. Res., 114, D21101.

Hofstra, N., New, M., McSweeney, 2010. The influence of interpolation and station network density on the distributions and trends of climate variables in gridded daily data C. Clim Dyn 35: 841.

Hutchinson, M.F., 1997. ANUSPLIN Versión 3.2 Disponible en: http://cres.anu.edu.au/software/ anusplin.html (Consulta: 18.10.2017).

Janatian, N., Sadeghi, M., Sanaeinejad, S.H., Bakhshian, E., Farid, A., Hasheminia, S.M. and Ghazanfari, S., 2017. A statistical framework for estimating air temperature using MODIS land surface temperature data. Int. J. Climatol., 37, pp. 1181-1194. doi:10.1002/joc.4766.

Kilibarda, M., Hengl, T., Heuvelink, G.B.M., Gräler, B., Pebesma, E., Per ec Tadić, M., Bajat B., 2014. Spatio-temporal interpolation of daily temperatures for global land areas at $1 \mathrm{~km}$ resolution, J. Geophys. Res. Atmos., 119, pp. 2294-2313.

Klene, A.E., Nelson, F.E., Shiklomanov, N.I., 2001. The N-factor in Natural Landscapes: Variability of Air and Soil-Surface Temperatures, Kuparuk River Basin, Alaska, USA., Arctic and Alpine Research, 33, pp. 140-148.

Landa, R., Magaña, V., Neri, C., 2008. Agua y clima: elementos para la adaptación al cambio climático. Semarnat, № 363.7387 , L3.

Lorenz, E., 1963. Deterministic no periodic flow. Journal of the Atmospheric Sciences, Vol. 20, March, Massachusetts, Institute of Technology.

Mildrexler, D.J., Zhao, M., Running, S.W., 2011. A global comparison between station air temperatures and MODIS land surface temperatures reveals the cooling role of forests, J. Geophys. Res., 116, G03025.

McLeman, R., 2013. Developments in modelling of climate change-related migration. Climatic Change, 117(3), pp. 599-611.

Moreno, A., Hasenauer, H., 2016. Spatial downscaling of European climate data. Int.J. Climatol., 36, pp. 1444-1458.

Mücher, C.A., Klijn, J.A., Wascher, D.M., Schaminée, J.H.J., 2010. A new European Landscape Classification (LANMAP): A transparent, flexible and user-oriented methodology to distinguish landscapes. Ecol. Indicat., 10 (2010), pp. 87-103.

Neteler, M., Metz, M., 2014. MODIS and vector-borne diseases. Special issue Earth Observation for Health IEEE Earthzine, Vol. 4.

Neteler, M., 2010. Estimating Daily Land Surface Temperatures in mountainous environments by reconstructed MODIS LST Data. Remote Sens. 2, pp. 333-351.

Patz, J.A., Campbell-Lendrum, D., Holloway, T., Foley, J.A., 2005. Impact of regional climate change on human health. Nature, 438(7066), pp. 310-317.

Peón, J., Recondo, C., Fernandez Calleja, J., 2014. Improvements in the estimation of daily minimum air temperature in peninsular Spain using MODIS land surface temperature. International Journal of Remote Sensing, 35, pp. 5148-5166. 10.1080/01431161.2014.935831. 
Ríos-Muñoz, C.A., Navarro-Sigüenza, A.G., 2009. Efectos del cambio de uso de suelo en la disponibilidad hipotética de hábitat para los psitácidos de México. Ornitología Neotropical, 20, pp. 491-509.

Rochlin, I., Ninivaggi, D.V., Hutchinson, M.L., Farajollahi, A. 2013. Climate change and range expansion of the Asian tiger mosquito (Aedes albopictus) in Northeastern USA: implications for public health practitioners. PloS one, 8(4), e60874.

Stern, N., 2006. Informe Stern: La economía del cambio climático. Cambridge, Reino Unido.

Salinger, M.J., 2005. Climate variability and change: past, present and future-an overview. Climatic Change, 70(1-2), pp. 9-29.

Sanchez-Lorenzo, A., Calbó, J., Martin-Vide, J., 2008. Spatial and temporal trends in sunshine duration over Western Europe (1938-2004). Journal of Climate, 21(22), pp. 6089-6098.

Scharlemann, J P., Benz, D., Hay, S.I., Purse, B.V., Tatem, A.J., Wint, G.W., Rogers, D.J., 2008. Global data for ecology and epidemiology: a novel algorithm for temporal Fourier processing MODIS data. PloS one, 3(1), e1408.

Sterling, S.M., Ducharne, A., Polcher, J., 2013. The impact of global land-cover change on the terrestrial water cycle. Nature Climate Change, 3(4), pp. 385-390.

Stewart, S.B., Nitschke, C.R., 2016, Improving temperature interpolation using MODIS LST and local topography: a comparison of methods in south east Australia. Int. J. Climatol., doi: 10.1002/joc. 4902 .

Sun, Y.-J., Wang, J.-F., Zhang, R.-H., Gillies, R.R., Xule, Y., Bo, Y.-C. (2005): "Air temperature retrieval from remote sensing data based on themodynamics", Theoretical and applied climatology, 80, pp. 37-48.

Liu S., J. Tian, H. Su, Y. Wu, 2016. Evaluating four remote sensing methods for estimating surface air temperature on a regional scale. American Meteorological. Society, doi: 10.1175/JAMCD-16-0188.1.

Van Doninck, J., De Baets, B., Peters, J., Hendrickx, G., Ducheyne, E., Verhoest, N.E., 2014. Modelling the Spatial Distribution of Culicoides imicola: Climatic versus Remote Sensing Data. Remote Sens., 6, 6604-6619.

Van den Besselaar, E.J.M., Haylock, M.R., van der Schrier, G., Klein Tank, A.M.G., 2011. A European daily high-resolution observational gridded data set of sea level pressure, J. Geophys. Res., 116, D11110.

Van der Schrier, G., van den Besselaar, E.J.M., Klein Tank, A.M.G., Verver, G., 2013. Monitoring European average temperature based on the E-OBS gridded data set, J. Geophys. Res. Atmos., 118, pp. 5120-5135.

Walther, G.R., Post, E., Convey, P., Menzel, A., Parmesan, C., Beebee, T. J., Bairlein, F., 2002. Ecological responses to recent climate change. Nature, 416(6879), pp. 389-395.

Westermann, S., Langer, M., Boike, J., 2011. Spatial and temporal variations of summer surface temperatures of high-arctic tundra on Svalbard - Implications for MODIS LST based permafrost monitoring, Remote Sens. Environ., 115, pp. 908-922.

Wan, Z., Zhang, Y., Zhang, Q., Li Z.-L., 2004. Quality assessment and validation of the MODIS global land surface temperature, Int. J. Remote Sens., 25, pp. 261-274. 
Wan, Z., Li, Z-L. 2008. Radiance-based validation of the V5 MODIS land-surface temperature product, Int. J. Remote Sens., 29, pp. 5373-5395.

Wan, Z., 2007. MODIS Land Surface Temperature products user's guide, Institute for Computational Earth System Science, University of California, Santa Barbara, CA, Disponible en: http:// www.icess.ucsb.edu/modis/LstUsrGuide/ MODIS LST products Users guide C5.pdf, (último acceso: 27 November 2016).

Zhao, M., Heinsch, F.A., Nemani, R.R., Running, S.W., 2005. Improvements of the MODIS terrestrial gross and net primary production global data set. Remote sensing of Environment, 95(2), pp. 164-176.

Zaksek, K., Schroedter-Homscheidt, M., 2009. Parameterization of air temperature in high temporal and spatial resolution from a combination of SEVIRI and MODIS instruments. ISPRS J. Photogramm., 4, pp. 414-421. 